\title{
Massive Black Hole Mergers
}

\author{
Enrico Barausse * \& Andrea Lapi
}

\begin{abstract}
At low redshift, massive black holes are found in the centers of almost all large elliptical galaxies, and also in many lower-mass systems. Their evolution is believed to be inextricably entangled with that of their host galaxies. On the one hand, the galactic environment provides gas for the black holes to grow via accretion and shine as active galactic nuclei. On the other hand, massive black holes are expected to backreact on the galactic dynamics, by injecting energy in their surroundings via jets or radiative feedback. Moreover, if galaxies and dark-matter halos form hierarchically, from small systems at high redshift coalescing into larger ones at more recent epochs, massive black holes may also merge, potentially generating gravitational-wave signals detectable by present and future experiments. In this Chapter, we discuss the predictions of current astrophysical models for the mergers of massive black holes in the $\mathrm{mHz}$ frequency band of the Laser Interferometer Space Antenna (LISA) and in the $\mathrm{nHz}$ frequency band of pulsar-timing array experiments. We focus in particular on the astrophysical uncertainties affecting these predictions, including the poorly known dynamical evolution of massive black hole pairs at separations of hundreds of parsecs; the possible formation of "stalled" binaries at parsec separations ("final-parsec problem"); and the effect of baryonic physics (e.g. SN feedback) on the growth of massive black holes. We show that nHz-band predic-

Enrico Barausse

SISSA - Scuola Internazionale Superiore di Studi Avanzati, Via Bonomea 265, 34135 Trieste, Italy IFPU - Institute for Fundamental Physics of the Universe, Via Beirut 2, 34014 Trieste, Italy INFN-Sezione di Trieste, via Valerio 2, 34127 Trieste, Italy

e-mail: baraussedsissa.it

Andrea Lapi

SISSA - Scuola Internazionale Superiore di Studi Avanzati, Via Bonomea 265, 34135 Trieste, Italy IFPU - Institute for Fundamental Physics of the Universe, Via Beirut 2, 34014 Trieste, Italy

INFN-Sezione di Trieste, via Valerio 2, 34127 Trieste, Italy

INAF-Osservatorio Astronomico di Trieste, via Tiepolo 11, 34131 Trieste, Italy

e-mail: lapiesissa.it

* corresponding author
\end{abstract}


tions are much more robust than in the $\mathrm{mHz}$ band, and comment on the implications of this fact for LISA and pulsar-timing arrays.

\section{Keywords}

black holes - gravitational waves — dark matter halos — galaxies: formation and evolution — LISA — pulsar-timing arrays

\section{Introduction}

In the local universe, massive black holes (MBHs) with masses of $10^{5}-10^{9} M_{\odot}$ are ubiquitous in the center of large elliptical galaxies [55, 73], and are also present in some low-mass dwarf galaxies [110, 111, 5]. MBHs are also believed to accrete from the nuclear gas and thus power Active Galactic Nuclei (AGNs) and quasars [53], which are in turn expected to exert a feedback (via jets or radiation) on their surroundings and even on their galactic host as a whole [33, 65, 24]. As a result, the evolution of MBHs and galaxies is expected to proceed "synergically", as suggested also by the observed galaxy-MBH scaling relations $[72,91,117,118$, 114, 115], which link e.g. the MBH mass to the stellar velocity dispersion of the host's spheroid ( $M_{\mathrm{bh}}-\sigma$ relation) and the MBH mass to the spheroid's stellar mass ( $M_{\mathrm{bh}}-M^{*}$ relation). (See however $[141,123,8,61]$ for recent work on these scaling relations, which shows that their interpretation may be subtler and at least partially related to observational bias.)

Despite their crucial importance for galaxy formation and evolution, the mechanisms that drive the growth, feedback, and dynamics of MBHs are surprisingly little understood. From a theoretical prospect, this is to be ascribed to the difficulty of resolving the scale of MBHs and their sphere of influence, which is tiny compared to galactic scales. Moreover, processes such as star formation and supernova (SN) explosions, but also MBH accretion, feedback and generally the interaction of MBHs with the surrounding gas are not yet fully understood from first principles and have therefore to be modeled via "sub-grid" prescriptions in hydrodynamic simulations (cf. e.g. [40, 46, 140, 116, 143, 131, 105, 100, 112]).

From an observational point of view, a crucial limitation consists in the difficulty of observing MBHs at moderate to high redshift, where their AGN/quasar activity is expected to be the brightest and their evolution the most rapid. Compared to electromagnetic probes, gravitational waves (GWs) offer several advantages in this respect (cf. Chapter 1). GWs interact very weakly with matter, unlike electromagnetic radiation. Moreover, GW detectors are sensitive not to the energy flux (which decays as the inverse square of the luminosity distance $d_{L}$ ) but to the field (which decays as

$d_{L}^{-1}$ ). For these reasons, GWs are in principle observable up to very high redshift. GWs, unlike electromagnetic radiation, are also not produced microscopically, i.e. 
they are not the collection of a huge number of quanta produced by atoms/plasmas. Instead, they are produced macroscropically by the bulk motion of large masses moving at relativistic speeds. As such, they encode directly information on the dynamics and macroscopic properties of the system that generates them. For GWs resulting from the merger of two black holes, one can e.g. extract the masses and spins of the two objects from the GW signal alone (cf. Chapter 43).

Dark-matter (DM) halos and galaxies are believed to form hierarchically in the $\Lambda$ Cold Dark Matter $(\Lambda \mathrm{CDM})$ model, from small systems at high redshift, which evolve to larger ones at lower redshift through a combination of major and minor mergers, as well as accretion of DM/hot gas from the intergalactic medium (IGM). It was therefore recognized early on [11] that galaxy mergers are a promising environment for the production of GWs, because they could lead to the coalescence of the MBHs present at the centers of the two merging galaxies. Because of the large MBH masses, these black-hole mergers are not observable with current interferometers such as LIGO and Virgo (cf. Chapter 1). Indeed, the merger frequency of a black-hole binary system of total mass $M$ scales roughly as $\sim 1 /\left(G M / c^{3}\right)$, which lies in the band $\left[10^{-4} \mathrm{~Hz}, 0.1 \mathrm{~Hz}\right]$ of the Laser Interferometer Space Antenna (LISA; cf. Chapter 3) for masses $10^{4}-10^{7} M_{\odot}$, and in the band $\left[10^{-9} \mathrm{~Hz}, 10^{-7} \mathrm{~Hz}\right]$ of pulsartiming arrays (PTAs; cf. Chapter 4 ) for masses of $10^{8}-10^{9} M_{\odot}$. .

In this Chapter, we will review the astrophysics of the co-eval evolution of MBHs and their galactic hosts. We will start from the large scales (i.e. $\lesssim 100 \mathrm{kpc}$ ) of DM halos and the diffuse, chemically pristine intergalactic medium, whose properties and merger history we will derive in detail within the framework of the $\Lambda$ CDM model. We will then focus on the intermediate scales (tens of kpcs down to pc) relevant for the baryonic physics of galaxies (e.g. stellar and gaseous disks and spheroids), down to the sub-pc scales of nuclear objects (nuclear star clusters and MBHs). We will then proceed to discuss the formation and evolution of MBHs (isolated and in binaries), starting from the formation of MBH pairs, which may then give rise to bound binaries and eventually merge. We will discuss the uncertainties of this "pairing" phase of the MBH evolution, and present its implications for LISA and PTAs.

Throughout this Chapter, we adopt the standard flat $\Lambda$ CDM cosmology [104] with rounded parameter values: fractional matter density at present time $\Omega_{M} \approx 0.3$, dark-energy density $\Omega_{\Lambda} \approx 0.7$, baryon density $\Omega_{\mathrm{b}} \approx 0.05$, Hubble constant $H_{0}=$ $100 h \mathrm{~km} \mathrm{~s}^{-1} \mathrm{Mpc}^{-1}$ with $h \approx 0.7$, and mass variance $\sigma_{8} \approx 0.8$ on a scale of $8 h^{-1}$ Mpc.

\section{Dark matter halos}

In the standard cosmological framework, the seeds of cosmic structures like quasars, galaxies, and galaxy systems are constituted by DM perturbations of the cosmic density field, originated by quantum effects during the early inflationary universe. The perturbations are amplified by gravitational instabilities and, as the local grav- 
ity prevails over the cosmic expansion, are enforced to collapse and virialize into bound "halos". In turn, these tend to grow hierarchically in mass and sequentially in time, with small clumps forming first and then stochastically merging together into larger and more massive objects. The halos provide the gravitational potential wells where baryonic matter can settle in virial equilibrium, and via several complex astrophysical processes (cooling, star formation, feedback, etc.) originate the luminous structures that populate the visible universe (see textbooks such as [29, 92]).

In the following, we recap, with a modern and original viewpoint, the crucial issues concerning the formation and evolution of DM halos, which will provide the backbone for the description of MBH mergers in the rest of this Chapter.

\section{Basic quantities}

Provided a background cosmology and suitable initial conditions in terms of a power spectrum $P(k)$ of density fluctuations (e.g., [10]), the statistical evolution of the halo populations in mass and redshift can be characterized at leading order via three basic quantities:

- The mass variance $\sigma(M)$, which describes the statistics of the density perturbation field when filtered on different mass scales $M$; this is defined as

$$
\sigma^{2}(M)=\frac{1}{(2 \pi)^{3}} \int \mathrm{d}^{3} k P(k) \tilde{W}_{M}^{2}(k),
$$

where $\tilde{W}_{M}^{2}(k)$ is the Fourier transform of a window function whose volume in real space encloses the mass $M$. For a scale-invariant power spectrum $P(k) \propto k^{n}$ with effective spectral index $n>-3$ (to ensure hierarchical clustering), $\sigma(M) \propto M^{-(n+3) / 6}$ applies.

- The linear threshold for collapse $\delta_{c}(z)$, which is a measure of the typical amplitude required for a perturbation to collapse efficiently; in fact, the condition $\sigma(M) \sim \delta_{c}(z)$ yields the characteristic mass $M_{c}(z)$ that, on statistical grounds, is prone to collapse at redshift $z$. A useful approximated expression for the collapse threshold in a flat $\Lambda \mathrm{CDM}$ cosmology is given by $\delta_{c}(z)=\delta_{c 0} D(0) / D(z)$, in terms of the normalization [49]

$$
\delta_{c 0} \simeq \frac{3}{20}(12 \pi)^{2 / 3}\left[1+0.0123 \log _{10} \Omega_{M}(z)\right] \approx 1.68,
$$

and of the growth factor [103]

$$
D(z) \simeq \frac{5}{2} \frac{\Omega_{M}(z)}{1+z}\left[\frac{1}{70}+\frac{209}{140} \Omega_{M}(z)-\frac{1}{140} \Omega_{M}^{2}(z)+\Omega_{M}^{4 / 7}(z)\right]^{-1}
$$




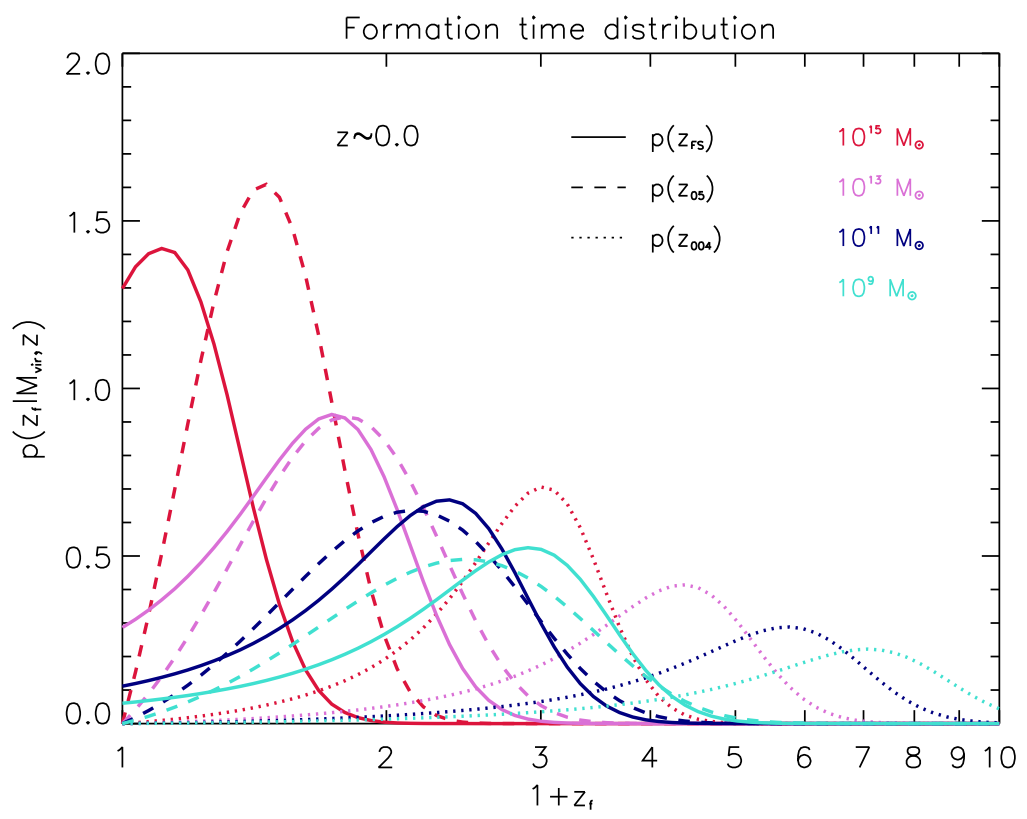

Fig. 1 Formation time distribution for different halo virial masses $M_{\mathrm{vir}} \approx 10^{15} M_{\odot}\left(\right.$ red), $\approx 10^{13} M_{\odot}$ (orchid), $\approx 10^{11} M_{\odot}$ (blue), and $\approx 10^{9} M_{\odot}$ (cyan) at current redshift $z \approx 0$. Dotted lines refer to the redshift $z_{0.04}$ at which the halo has accumulated $4 \%$ of its current mass, dashed lines to the redshift $z_{0.5}$ at which the halo has accumulated half of its current mass, and solid lines to the redshift $z_{\mathrm{FS}}$ of the transition between fast collapse and slow accretion.

where $\Omega_{M}(z)=\Omega_{M}(1+z)^{3} /\left[\Omega_{\Lambda}+\Omega_{M}(1+z)^{3}\right]$; this latter redshift dependence is close to $D(z) \propto(1+z)^{-1}$ at $z \gtrsim 1$, and then slows down toward the present when the dark energy component kicks in.

- The virial overdensity $\Delta_{\mathrm{vir}}$, which approximately renders the typical density contrast of perturbations at virialization (hence in the nonlinear regime). In a flat $\Lambda \mathrm{CDM}$ cosmology it can be computed via the approximated formula [26]

$$
\Delta_{\mathrm{vir}} \approx 18 \pi^{2}+82\left[\Omega_{M}(z)-1\right]-39\left[\Omega_{M}(z)-1\right]^{2},
$$

with typical values $\Delta_{\mathrm{vir}} \approx 100$ at the present time and increasing toward $\Delta_{\mathrm{vir}} \approx 180$ for $z \gtrsim 1$. Related to this, the virial mass $M_{\text {vir }}$ of a DM halo is defined as the mass contained within a radius $R_{\mathrm{vir}}$ inside which the mean interior density is $\Delta_{\mathrm{vir}}$ times the critical density $\rho_{\text {crit }} \equiv 3 H_{0}^{2} / 8 \pi G \approx 2.8 \times 10^{11} h^{2} M_{\odot} \mathrm{Mpc}^{-3}$. 
Formation time, fast collapse vs. slow accretion

The formation redshift $z_{f}$ of a halo with mass $M$ at redshift $z$ is routinely defined as the highest redshift at which the mass of its main progenitor is larger than $f M$, i.e. a fraction $f$ of the current mass. [58] have provided a fitting formula of the formation redshift distribution extracted by $N$-body simulations, valid for any value of $f$. It writes

$$
\frac{\mathrm{d} p_{f}}{\mathrm{~d} z_{f}}\left(z_{f} \mid M, z\right)=\frac{\alpha_{f} v_{f} e^{v_{f}^{2} / 2}}{\left(e^{v_{f}^{2} / 2}+\alpha_{f}-1\right)^{2}}\left|\frac{\mathrm{d} v_{f}}{\mathrm{~d} z_{f}}\right|
$$

where $\alpha_{f} \approx 0.815 e^{-2 f^{3}} / f^{0.707}$ and

$$
v_{f} \equiv \frac{\delta_{c}\left(z_{f}\right)-\delta_{c}(z)}{\sqrt{\sigma^{2}(f M)-\sigma^{2}(M)}} .
$$

The corresponding cumulative distribution is

$$
p_{f}\left(>z_{f} \mid M, z\right)=\frac{\alpha_{f}}{e^{v_{f}^{2} / 2}+\alpha_{f}-1} .
$$

Despite $f \approx 1 / 2$ is often exploited to define the formation redshift of a halo, this choice is rather arbitrary. In fact, intensive $N$-body simulations and analytic studies (see $[148,149,77]$ ) have demonstrated that the growth of a halo actually comprises two different regimes: an early fast collapse during which the central gravitational potential well is built up by dynamical relaxation processes; and a late slow accretion when mass is slowly added in the outskirts of the halo in the way of an inside out growth. The transition between the fast and slow accretion regime is a more motivated definition of halo formation; this is found to occur at a cosmic time $t_{z_{\mathrm{FS}}} \approx 3.75 t_{z_{0.04}}$, proportional to that at which the halo assembled a fraction $f \approx 4 \%$ of its current mass. Thus, the generic formation time distribution $\mathrm{d} p / \mathrm{d} z_{f}$ in Eq. (5) can be exploited to derive the distributions $\mathrm{d} p / \mathrm{d} z_{0.04}$ and $\mathrm{d} p / \mathrm{d} z_{\mathrm{FS}}$. Note that the latter can in principle extend even to redshifts $z_{\mathrm{FS}}<z$, meaning that a halo at redshift $z$ has not yet entered the slow accretion regime. The corresponding distributions for different halo virial masses $M_{\mathrm{vir}} \approx 10^{9}-10^{15} M_{\odot}$ at $z \approx 0$ are illustrated in Fig. 1 .

\section{Median and average halo mass growth}

The median history of the main progenitor for a halo with mass $M$ at redshift $z$ can be derived very easily from the formation redshift distribution Eq. (5). By definition, if $\mathrm{d} p_{f} / \mathrm{d} z_{f}$ is the formation redshift distribution, and $\mathrm{d} p_{\mathrm{MP}} / \mathrm{d} M^{\prime}$ is the distribution of main progenitor masses, one has the trivial identity 


$$
\int_{z_{f}}^{\infty} \mathrm{d} z^{\prime} \frac{\mathrm{d} p_{f}}{\mathrm{~d} z^{\prime}}\left(z^{\prime} \mid M, z\right)=\int_{f M}^{M} \mathrm{~d} M^{\prime} \frac{\mathrm{d} p_{M P}}{\mathrm{~d} M^{\prime}}\left(M^{\prime}, z_{f} \mid M, z\right) .
$$

From this it is easily recognized that the cumulative distributions of formation redshift and main progenitor masses are equal; thus, both the median main progenitor mass at given redshift and the median formation redshift at given main progenitor mass both satisfy Eq. (6), i.e.

$$
\left[\sigma^{2}(f M)-\sigma^{2}(M)\right] \tilde{v}_{f}^{2}=\left[\delta_{c}\left(z^{\prime}\right)-\delta_{c}(z)\right]^{2}
$$

where

$$
\tilde{v}_{f}=\sqrt{2 \ln \left(1+\alpha_{f}\right)}
$$

is the median value that has been computed explicitly from Eq. (7). The above equation can be solved for the median $\tilde{f}\left(z^{\prime}\right)$ and hence the main progenitor median mass is $\tilde{f}\left(z^{\prime}\right) M$.

The average mass growth of the main progenitor $\langle f\rangle M$ can be related to the (cumulative) formation time distribution. By definition one has

$$
\left\langle f\left(z^{\prime}\right)\right\rangle M=\int_{0}^{M} \mathrm{~d} M^{\prime} M^{\prime} \frac{\mathrm{d} p_{\mathrm{MP}}}{\mathrm{d} M^{\prime}}\left(M^{\prime}, z^{\prime} \mid M, z\right)
$$

now one can rewrite $M^{\prime}=\int_{0}^{M^{\prime}} \mathrm{d} M^{\prime \prime}$, reverse the double (triangular) integral as $\int_{0}^{M} \mathrm{~d} M^{\prime} \int_{0}^{M^{\prime}} \mathrm{d} M^{\prime \prime}=\int_{0}^{M} \mathrm{~d} M^{\prime \prime} \int_{M^{\prime \prime}}^{M} \mathrm{~d} M^{\prime}$, and use Eq. (8) to obtain

$$
\left\langle f\left(z^{\prime}\right)\right\rangle M=M \int_{0}^{1} \mathrm{~d} f p_{f}\left(>z^{\prime} \mid M, z\right)
$$

Notice that to compute this quantity the formation time distribution for any $f$ is necessary.

The corresponding median and average halo growth histories for different halo virial masses $M_{\text {vir }} \approx 10^{9}-10^{15} M_{\odot}$ at $z \approx 0$ are illustrated in Fig. 2 and found to be in overall good agreement with the tracks directly extracted from simulations [13].

\section{Radial mass profile and pseudo evolution}

$\mathrm{N}$-body simulations indicate that the mass profile of a DM halo follows the universal Navarro-Frenk-White (NFW) shape [99]

$$
M(<r)=M_{\Delta} g\left(c_{\Delta}\right)\left[\ln \left(1+c_{\Delta} s\right)-\frac{c_{\Delta} s}{1+c_{\Delta} s}\right]
$$

where $s \equiv r / R_{\Delta}$ is the radius normalized to that $R_{\Delta} \equiv\left(M_{\Delta} / 4 \pi \Delta \rho_{\text {crit }}\right)^{1 / 3}$ within which the average density is $\Delta$ times the critical one $\rho_{\text {crit }} \approx 2.8 \times 10^{11} \Omega_{M} h^{2} M_{\odot}$ 


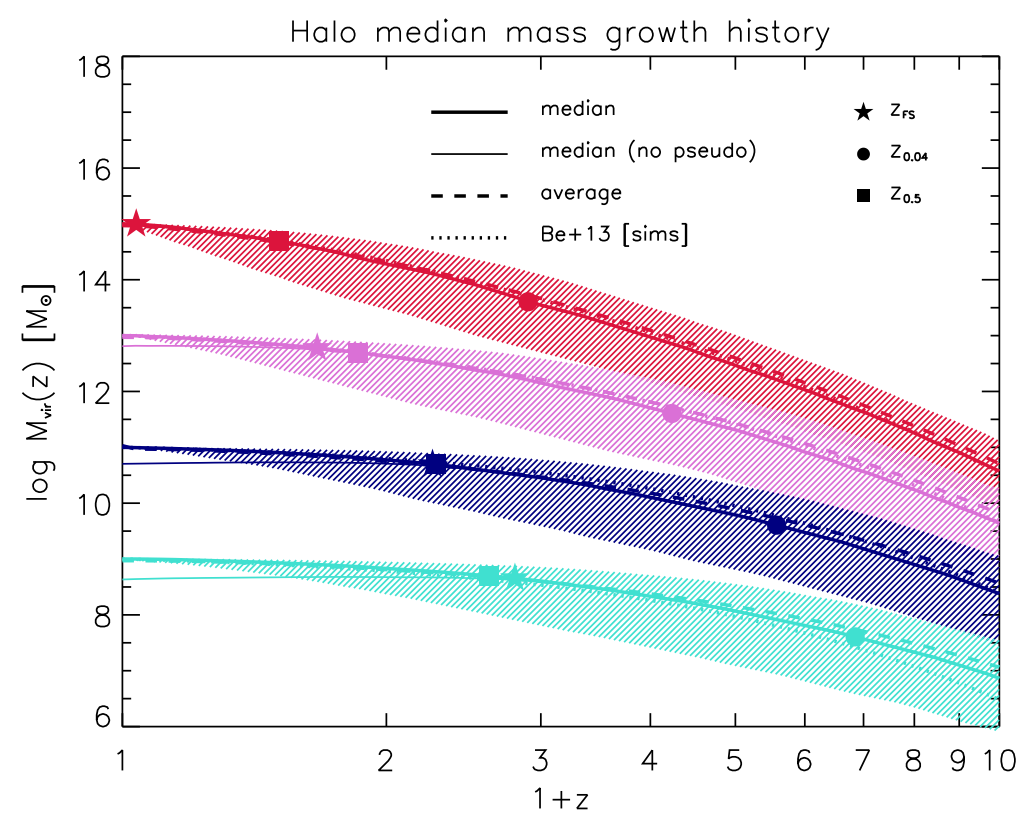

Fig. 2 Mass growth history of the main progenitor for halos with different virial masses $M_{\text {vir }} \approx$ $10^{15} M_{\odot}($ red $), \approx 10^{13} M_{\odot}($ orchid $), \approx 10^{11} M_{\odot}$ (blue), and $\approx 10^{9} M_{\odot}$ (cyan) at current redshift $z \approx 0$. Thick solid lines refer to the median growth history, and shaded areas illustrate 5 th and 95 th percentile around it. Thin solid lines refer to the median history depurated from pseudo-evolution, dashed lines to the average growth history, and dotted lines show the direct simulation fits by [13]. The symbols illustrate on every median growth history the location of the redshift $z_{0.04}$ (circles) where the main progenitor mass is $4 \%$ of the current one, $z_{0.5}$ (squares) where it is $50 \%$ of the current one, and $z_{\mathrm{FS}}$ (stars) where transition from fast to slow accretion regimes takes place.

$\mathrm{Mpc}^{-3}$; moreover, $c_{\Delta}$ is the concentration parameter and $g(x) \equiv[\ln (1+x)-x /(1+$ $x)]^{-1}$. Very often $\Delta$ is taken to be the virial threshold for collapse $\Delta_{\text {vir }}$ defined in Eq. (4), so that the related concentration will be indicated by $c_{\mathrm{vir}}$.

Simulations indicate (see $[148,149,96,44]$ ) that during the fast collapse the virial concentration $c_{\text {vir }}$ stays put to a value around 4 , while it progressively increases as mass is added in the outer parts of the halo during the slow accretion phase. A simple fitting formula is given by [149]

$$
c_{\mathrm{vir}}\left(z^{\prime} \mid M_{\mathrm{vir}}, z\right) \approx 4\left[1+\left(t_{z^{\prime}} / t_{z_{\mathrm{FS}}}\right)^{8.4}\right]^{1 / 8}
$$

in terms of the fast/slow transition time $t_{\mathrm{FS}}\left(M_{\mathrm{vir}}, z\right)$; note that the latter depends on the halo virial mass $M_{\mathrm{vir}}$ at redshift $z$, and that actually is not a unique value but follows the distribution $\mathrm{d} p / \mathrm{d} z \mathrm{FS}$ defined above. The median concentration corresponding to the halo mass growth histories discussed above for different halo virial masses $M_{\mathrm{vir}} \approx 10^{9}-10^{15} M_{\odot}$ at $z \approx 0$ is shown in Fig. 3 . 
It has been pointed out by several authors (e.g., [43, 96, 44] that during the slow accretion phase the halo growth is mainly driven by a pseudo-evolution in radius and mass due to the lowering of the reference density defining the halo boundary. For example, pseudo-evolution implies that a halo of $10^{13} M_{\odot}$ with turning point at redshift $z_{\mathrm{FS}} \approx 2$ will end up, say, at $z \approx 0$ in a halo of several $10^{14} M_{\odot}$; clearly the latter is a halo typical of a galaxy group/cluster, but has little relevance to the physical processes occurring within the galaxy hosted by the original halo of $10^{13} M_{\odot}$ at

formation. In other words, the 'galactic' halo must have evolved in mass much less than predicted by pseudo-evolution. Under the assumption that the universal NFW mass distribution is retained, the mass growth depurated from pseudo-evolution can be estimated as $[96,44]$

$$
M_{\text {nops }}(z) \simeq M_{\mathrm{vir}}(z) g\left[c_{\mathrm{vir}}(z)\right] / g\left[c_{\mathrm{vir}}\left(z_{\mathrm{FS}}\right)\right]
$$

This also implies that the overdensity of the central region not subject to pseudoevolution is given by

$$
\Delta_{\text {nops }}(z) \simeq \Delta_{\text {vir }}\left[\frac{c_{\text {vir }}(z)}{c_{\mathrm{vir}}\left(z_{\mathrm{FS}}\right)}\right]^{3} \frac{g\left[c_{\mathrm{vir}}(z)\right]}{g\left[c_{\mathrm{vir}}\left(z_{\mathrm{FS}}\right)\right]}
$$

We have highlighted in Figs. 2 and 3 the impact of the pseudo-evolution on the mass growth and on the concentration/central region overdensity.

\section{Halo spin}

DM halos slowly rotate with a specific (i.e., per unit mass) angular momentum given by

$$
j_{\mathrm{H}} \approx 1670 \frac{\lambda}{0.035}\left(\frac{M_{\mathrm{H}}}{10^{12} M_{\odot}}\right)^{2 / 3} E_{z}^{-1 / 6} \mathrm{~km} \mathrm{~s}^{-1} \mathrm{kpc},
$$

where $E_{z} \equiv \Omega_{\Lambda}+\Omega_{M}(1+z)^{3}$ and $\lambda$ is the halo spin parameter. Numerical simulations (e.g., [27, 84, 150]) have found that $\lambda$ has a roughly log-normal distribution with mean value $\langle\lambda\rangle \approx 0.035$ and dispersion $\sigma_{\log \lambda} \approx 0.25 \mathrm{dex}$, approximately independent of mass and redshift. The small values of $\lambda$ testify that rotation is largely subdominant with respect to random motions, which indeed are mainly responsible for sustaining gravity and enforcing virial equilibrium.

The distribution of DM halo mass $M_{\mathrm{H}}(<j)$ as a function of the specific angular momentum $j$ has been studied in detail via $N$-body simulations by [27, 133, 125], who found the convenient one-parameter representation

$$
M_{\mathrm{H}}(<j)=M_{\mathrm{H}} \gamma\left[\alpha, \alpha j / j_{\mathrm{H}}\right]
$$

where $\gamma[a, x] \equiv \int_{0}^{x} \mathrm{~d} t t^{a-1} e^{-t} / \int_{0}^{\infty} \mathrm{d} t t^{a-1} e^{-t}$ is the normalized incomplete gamma function, and the value $\alpha \approx 0.9$ applies. All in all, a representation in spherical mass 


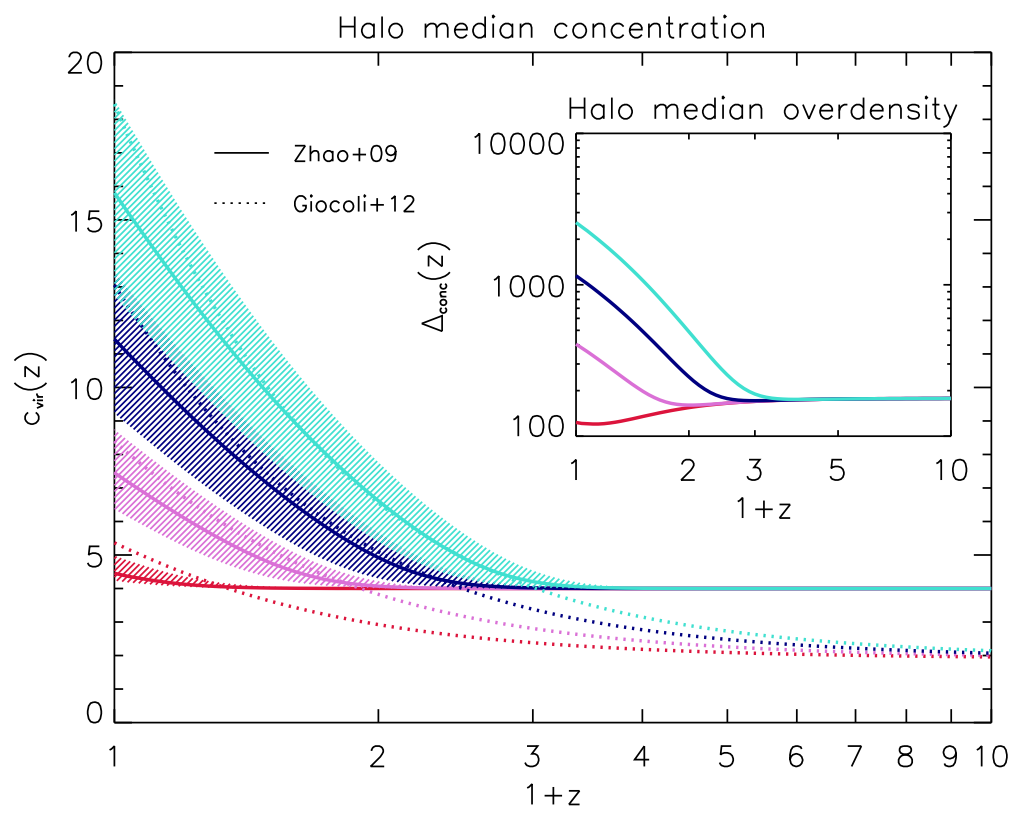

Fig. 3 Median virial concentration of halos with different halo virial masses $M_{\mathrm{vir}} \approx 10^{15} M_{\odot}$ (red), $\approx 10^{13} M_{\odot}$ (orchid), $\approx 10^{11} M_{\odot}$ (blue), and $\approx 10^{9} M_{\odot}$ (cyan) at current redshift $z \approx 0$; colored shaded area illustrate 5 th and 95 th percentile around the median. Solid lines refer to the prescription by [149] and dotted lines to that by [58]. The inset shows the overdensity $\Delta_{\text {conc }}$ of the inner region formed during the fast collapse phase.

shells $j \propto M(<r)^{s}$ with $s \sim 1.1-1.3$ holds to a very good approximation over an extended range.

\section{Halo mass function}

The halo mass function is the statistics describing the number of DM halos per unit comoving volume as a function of halo mass and redshift. This is routinely estimated via high-resolution, large-volume $N$-body simulations (see [126, 66, 130, $15,144]$ ), although given the natural limits on resolution, computational time, and storing capacity, it can be probed only in limited mass and redshift ranges. We also caveat that the results of simulations depend somewhat on the algorithm used to identify collapsed halos (e.g., $\mathrm{FoF}=$ friend-of-friend vs. $\mathrm{SO}=$ spherical overdensity), and on specific parameters related to the identification of isolated objects (e.g., the linking length). 


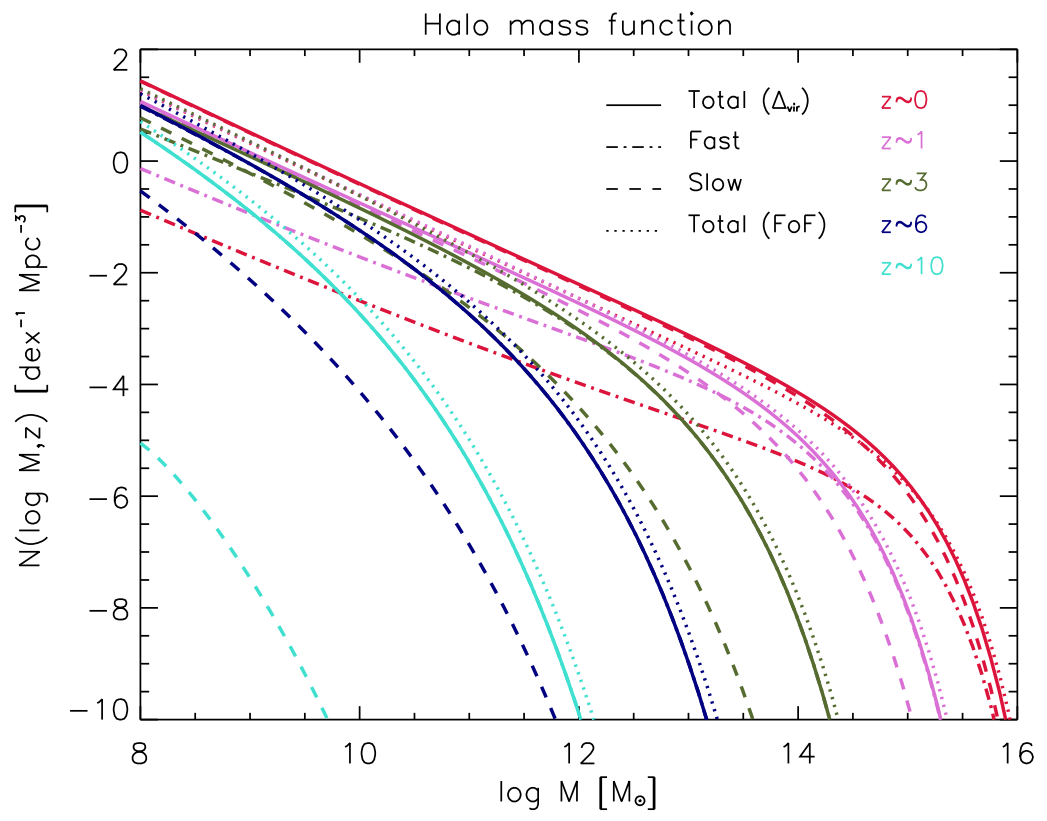

Fig. 4 Halo mass function $N(\log M, z) \equiv \mathrm{d} N / \mathrm{d} \log M$ per $\operatorname{logarithmic~mass~bins~(dex~units)~at~dif-~}$ ferent redshifts $z \approx 0$ (red), 1 (orchid), 3 (green), 6 (blue) and 10 (turquoise). Solid line is the total mass function by [144] for a nonlinear threshold $\Delta_{\mathrm{vir}}$, dot-dashed line is the contribution from halos in the fast collapse stage, and dashed line from those in the slow accretion regime. Dotted lines illustrate the mass function for FoF-identified halos.

One of the most accurate determination has been obtained by [144]. Their fit to the simulation outcomes reads

$$
\frac{\mathrm{d} N}{\mathrm{~d} M}=\frac{\rho_{M}(0)}{M^{2}}\left|\frac{\mathrm{d} \log \sigma}{\mathrm{d} \log M}\right| \Gamma(\sigma, z \mid \Delta) f(\sigma, z \mid \Delta)
$$

where

$$
f(\sigma, z \mid \Delta)=A\left[1+\left(\frac{b}{\sigma}\right)^{a}\right] e^{-c / \sigma^{2}}
$$

For FoF-identified halos the function $\Gamma(\sigma, z \mid \Delta)=1$ and the relevant parameters read $A=0.282, \alpha=2.163, \beta=1.406$ and $\gamma=1.210$; thus the function $f(\sigma)$ is actually independent of the redshift and the mass function is said to be universal. For halos identified with a spherical overdensity algorithm, one has

$$
\Gamma(\sigma, z \mid \Delta)=C(\Delta)\left(\frac{\Delta}{178}\right)^{d(z)} e^{p(1-\Delta / 178) / \sigma^{q}}
$$


and redshift-dependent parameters $A=\Omega_{M}(z)\left[1.907(1+z)^{-3.216}+0.074\right], a=$ $\Omega_{M}(z)\left[3.136(1+z)^{-3.058}+2.349\right], b=\Omega_{M}(z)\left[5.907(1+z)^{-3.599}+2.344\right], c=$ $1.318, C(\Delta)=0.947 e^{0.023(\Delta / 178-1)}, d(z)=-0.456 \Omega_{M}(z)-0.139, p=0.072$ and $q=2.130$. The halo mass function for both $\mathrm{FoF}$ and $\mathrm{SO}$ algorithms is illustrated in Fig. 4, together with the contribution from halo in the fast and slow accretion regime.

The shape of the mass function and other halo statistics (like progenitor mass function and large-scale bias) can be theoretically understood from first principles, on the basis of the celebrated Press \& Schechter theory by [107] and its extended version by $[19,76,93]$. Such a framework, modernly dubbed excursion set theory, remaps the issue of counting numbers of halos into finding the first crossing distribution of a random walk that hits a suitable barrier. The random walk is executed by the overdensity field around a given spatial location when considered as a function of the mass variance $\sigma^{2}(M)$ of Eq. (1). The barrier is provided by the linear collapse threshold $\delta_{c}(t)$ in Eqs. (2-3), with possibly an additional dependence on the mass scale ('moving barrier'). Remarkably, the resulting halo statistics are found to be in overall good agreement with the $N$-body outcomes, especially when a moving barrier with shape inspired by the ellipsoidal collapse is adopted [126, 146, 147].

Recently, an alternative framework to describe the halo statistics based on stochastic differential equations in real space has been proposed by [78].

\section{Halo merger trees}

Halo merger trees are numerical yet approximate realizations of a halo merging history (see [68, 127, 31, 102]); these constitute the skeleton of many semi-analytic models aimed to describe the properties of galaxies and MBHs.

The basic ingredient to build up the tree is the halo progenitor mass function $\mathrm{d} N / \mathrm{d} M^{\prime}$, i.e., the distribution of halo masses $M^{\prime}$ at different redshifts $z^{\prime}$ that will end up in a given descendant halo mass $M$ at a later time $z$. This quantity is usually represented as

$$
\frac{\mathrm{d} N}{\mathrm{~d} M^{\prime}}\left(M^{\prime}, z^{\prime} \mid M, z\right) \simeq \frac{M}{M^{\prime}} \frac{\Delta \delta_{c} e^{-\left(\Delta \delta_{c}\right)^{2} / 2 \Delta \sigma^{2}}}{\sqrt{2 \pi}\left(\Delta \sigma^{2}\right)^{3 / 2}} G\left(1+\frac{\Delta \sigma^{2}}{\sigma^{2}}, \frac{\delta_{c}^{2}}{\sigma^{2}}\right)\left|\frac{\mathrm{d} \sigma^{2}}{\mathrm{~d} M}\right|_{M^{\prime}}
$$

in terms of $\Delta \delta_{c}=\delta_{c}\left(z^{\prime}\right)-\delta_{c}(z)$ and $\Delta \sigma^{2}=\sigma^{2}\left(M^{\prime}\right)-\sigma^{2}(M)$; actually this expression is inspired by the excursion set theory (see above), but includes a correction term to reproduce the results of N-body simulations [102, 67], that reads

$$
G\left(1+\frac{\Delta \sigma^{2}}{\sigma^{2}}, \frac{\delta_{c}^{2}}{\sigma^{2}}\right) \simeq G_{0}\left(1+\frac{\Delta \sigma^{2}}{\sigma^{2}}\right)^{\gamma_{1}}\left(\frac{\delta_{c}^{2}}{\sigma^{2}}\right)^{\gamma_{2}}
$$

with $G_{0} \approx 0.57, \gamma_{1} \approx 0.19$ and $\gamma_{2} \approx-0.005$. 


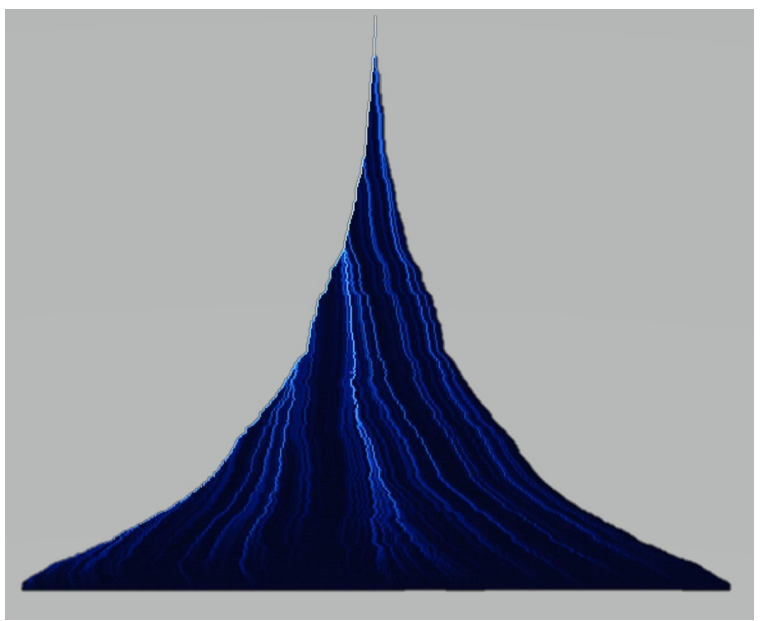

Fig. 5 Example of a merger tree for a descendant halo with final mass $10^{13} M_{\odot}$ at $z \approx 0$ (top), down to an initial redshift $z \sim 10$ (bottom); bluer colors correspond to more massive progenitors.

There are various possible algorithms to exploit the above and build up the tree, but one of the most effective in reproducing $N$-body outcomes is the [31] binary method with accretion, which is briefly recalled next. First, provided a mass resolution $M_{\text {res }}$, one computes the smooth mass accretion integrating the progenitor mass function

$$
M_{\mathrm{acc}} \simeq \int_{0}^{M_{\mathrm{res}}} \mathrm{d} M^{\prime} M^{\prime} \frac{\mathrm{d} N}{\mathrm{~d} M^{\prime}}\left(M^{\prime}, z^{\prime} \mid M, z\right) .
$$

Second, one computes the mean number of progenitors in the range $\left[M_{\text {res }}, M / 2\right]$ as

$$
\mathscr{P} \simeq \int_{M_{\mathrm{res}}}^{M / 2} \mathrm{~d} M^{\prime} \frac{\mathrm{d} N}{\mathrm{~d} M^{\prime}}\left(M^{\prime}, z^{\prime} \mid M, z\right),
$$

and chooses the merger time step so that $\mathscr{P} \ll 1$ to ensure that multiple fragmentation is unlikely. A uniform random number $\mathscr{R}$ generated in the interval $[0,1]$ determines whether the descendant has one $(\mathscr{R}>\mathscr{P})$ or two progenitors $(\mathscr{R} \leq \mathscr{P})$. In the former case, one prescribes $M^{\prime}=M-M_{\text {acc }}$; in the latter case, one of the masses $M_{1}^{\prime}$ is drawn from the progenitor mass function in the range $\left[M_{\text {res }}, M / 2\right]$ and the second mass is just $M_{2}^{\prime}=M-M_{1}^{\prime}-M_{\text {res }}$.

\section{Halo merger rates}

After formation, a DM halo is expected to grow because of mergers and smooth accretion from the surrounding field and the cosmic web. The merger rates per descendant halo, per unit cosmic time $t$ (corresponding to redshift $z$ ) and per halo mass 


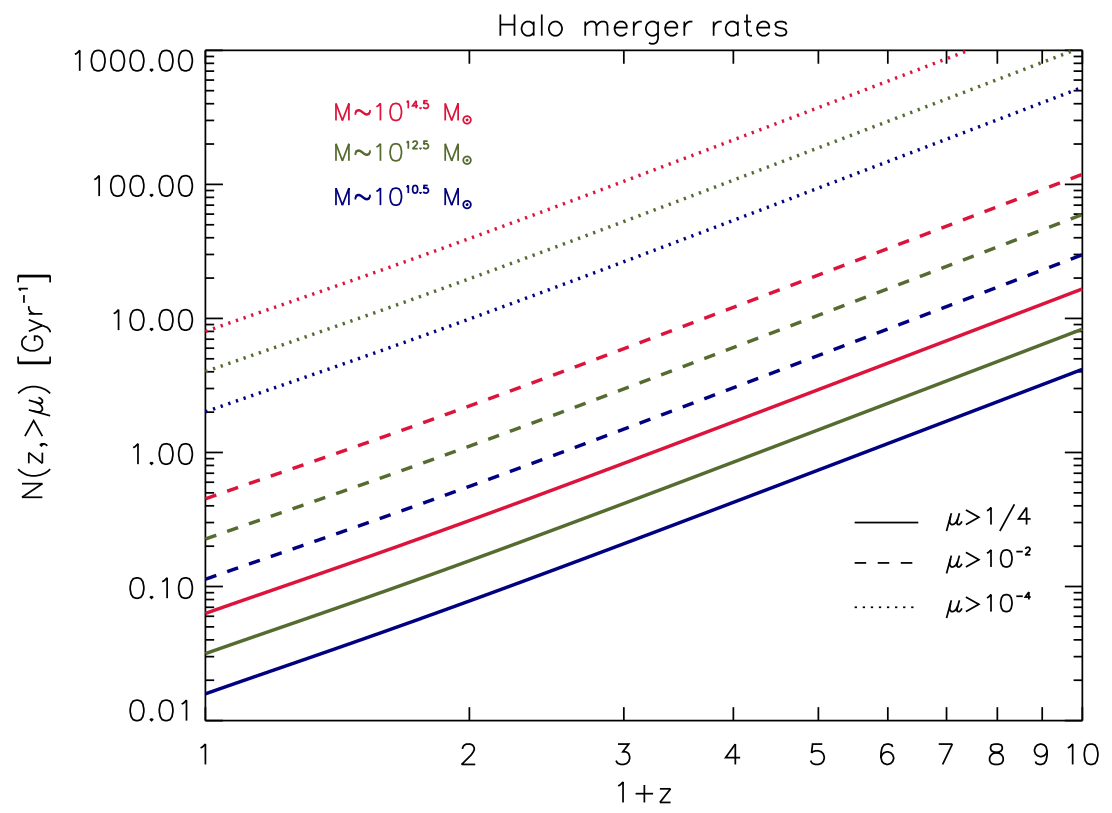

Fig. 6 Halo merger rate as a function of redshift for different halo masses $M_{\mathrm{H}} \approx 10^{14.5} M_{\odot}$ (red), $10^{12.5} M_{\odot}$ (green), $10^{10.5} M_{\odot}$ (blue), and different thresholds in halo mass ratios $\mu_{\mathrm{H}}>1 / 4$ (solid lines), $\mu_{\mathrm{H}}>10^{-2}$ (dashed lines), and $\mu_{\mathrm{H}}>10^{-4}$ (dotted lines).

ratio $\mu_{\mathrm{H}}$ can be described with the fitting formula originally proposed by $[50,79]$

$$
\frac{\mathrm{d} N_{\mathrm{H}, \text { merg }}}{\mathrm{d} t \mathrm{~d} \mu_{\mathrm{H}}}=N_{\mathrm{H}} M_{\mathrm{H}, 12}^{a} \mu_{\mathrm{H}}^{-b-2} e^{\left(\mu_{\mathrm{H}} / \tilde{\mu}_{\mathrm{H}}\right)^{c}} \frac{\mathrm{d} \delta_{c}}{\mathrm{~d} t}
$$

in terms of the descendant halo mass $M_{\mathrm{H}, 12}=M_{\mathrm{H}} / 10^{12} M_{\odot}$, and of the linear threshold for collapse $\delta_{c}$. [56] have determined the parameters entering the above expression from the Illustris-Dark simulations, finding $N_{\mathrm{H}}=0.065, a=0.15$, $b=-0.3, c=0.5$ and $\tilde{\mu}_{\mathrm{H}}=0.4$. Major mergers are typically identified with the events featuring $\mu_{\mathrm{H}}>1 / 4$, minor mergers with those featuring $1 / 10<\mu_{\mathrm{H}}<1 / 4$, and smooth accretion with those having $\mu_{\mathrm{H}}<1 / 100$.

The resulting halo merger rates as a function of the mass ratio for different redshifts are illustrated in Fig. 6. We stress that these are often exploited to derive the MBH merger rates, by taking into account possible time delays due to a variety of dynamical processes; these will be discussed to some extent in the rest of this Chapter. 


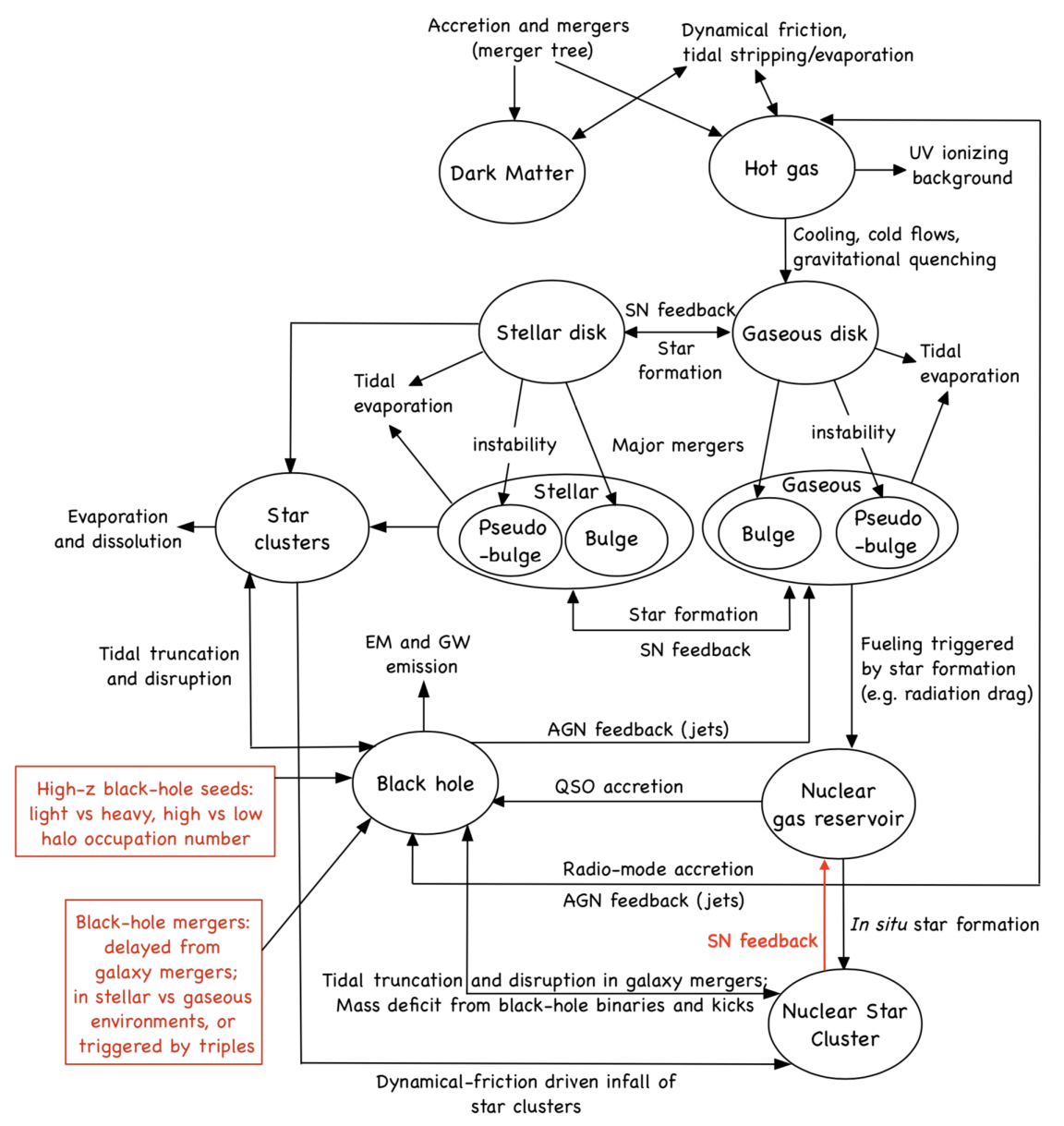

Fig. 7 Figure adapted from [71]. Schematic description of the semi-analytic model of [7], with the improvements of [121, 1, 22, 23, 9]. Highlighted in red are the processes (black-hole seeding, delays and $\mathrm{SN}$ feedback on the MBH growth) that most significantly affect MBH merger rates.

\section{Baryons and black holes}

The DM merger trees described above provide the backbone on top of which the hierarchical evolution of the baryonic structures of galaxies, as well as the MBHs, can be computed. In the following, we briefly describe the physics of baryons, before focusing on MBHs and presenting results for the $\mathrm{MBH}$ merger rates. We refer e.g. to [7] for a more extensive description of the physics. A schematic view of the various baryonic structures involved and their relations is presented in Fig. 7.

On the largest scales, baryons are mostly in the form of a chemically unprocessed gas that accretes onto DM halos from the IGM. While accreting, the gas is 
shock-heated to the virial temperature of the halo in low-redshift systems of sufficiently large mass. However, in smaller systems and/or at high redshift, the IGM may simply stream into the DM halo along cold flows/filaments [36, 28, 37]. (In practice, in simulations, a smooth transition is observed between these "hot" and "cold" accretion modes of the IGM, see e.g. [32].)

Cooling of the shock-heated gas and/or inflow of the IGM along cold filaments is then expected to give rise to a cold-gas phase ("interstellar medium", ISM). Because of conservation of angular momentum, this gaseous component may be disklike [94], although its geometry can be significantly altered and rendered spheroidal by bar instabilities, compaction and (major) mergers. Moreover, the ISM (and particularly its molecular clouds) will also undergo star formation. The latter will take place both in disks and in spheroids (or "bulges"), although possibly more efficiently in the latter [35, 57], since the influx of gas toward the galaxy's center as a result of major mergers and disk instabilities may trigger starbursts. Star formation will also exert a feedback on the stellar surroundings via SN explosions [128, 54, 109], whose winds are expected to expel gas from the ISM, thus quenching (or self-regulating) star formation. SN ejecta also enrich the metal content of the ISM and contribute to its chemical evolution.

In their central regions, small to intermediate local galaxies with bulge velocity dispersion $\sigma \lesssim 150 \mathrm{~km} / \mathrm{s}$ typically contain dense $(\sim 10 \mathrm{pc})$ stellar clusters with mass up to $10^{8} M_{\odot}$ [52]. These "nuclear star clusters" might form from local ("in situ") star formation episodes and/or infall of globular clusters from the galactic disk/bulge as a result of mass segregation (i.e. dynamical friction from the gas and field stars) $[2,1]$. Their evolution is intimately connected with that of MBHs, which also dwell in the same nuclear region [60,59]. The merger of two galaxies, and the ensuing formation of a MBH pair or bound binary, is likely responsible for the relative scarcity of nuclear star clusters in systems with $\sigma \gtrsim 150 \mathrm{~km} / \mathrm{s}[14,2,1]$. Indeed, because of the galaxy-MBH scaling relations, those large galaxies tend to host large MBHs. When the latter form pairs/binaries, they erode nuclear star clusters by ejecting stars through the slingshot effect. Moreover, MBH accretion occurs from the same nuclear gas (mainly resulting from inflows due to major mergers and/or disk instabilities) that feeds the growth of nuclear star clusters via in-situ star formation episodes. It is therefore natural to expect SN explosions, which tend to deplete/heat up the ISM, to impact also the amount of gas that can accrete onto the MBH. This effect is indeed observed in hydrodynamic simulations, though it depends sensitively on the details of how the energy released by SNae couples to the ISM. For instance, weak thermal and kinetic SN feedbacks do not dramatically impact $\mathrm{BH}$ growth, but if the shocks caused by the explosions delay cooling of the gas, SN feedback may hamper BH growth in low-mass galaxies [62].

Besides being passive actors in galaxy formation, with their mass growth and spin evolution proceeding through gas accretion and coalescences triggered by galaxy mergers, MBHs can also backreact on their galactic hosts. This process is known as AGN feedback [33, 65, 24]. When accreting and shining as quasars/AGNs, MBHs emit photons in a variety of bands. A fraction of this radiation may interact with the (optically thick) material surrounding MBHs, heating it up and thus quench- 
ing its cooling and star formation. Moreover, AGN feedback may be provided by outflows/jets from the accretion disk ("disk winds") [16], or by jets launched by the MBHs when they are highly spinning and immersed in an external magnetic field (anchored to a circum-nuclear disk). The latter process is known as BlandfordZnajek effect [17], is linked to the presence of a black-hole ergoregion, and is believed to be the mechanism underpinning radio-loud AGNs.

The baryonic physics that we have described is typically implemented in both semi-analytic galaxy-formation models (e.g. [69, 30, 31, 95, 7]) as well as in hydrodynamic simulations (e.g. [40, 46, 140, 116, 143, 131, 105, 62, 100, 112]). We will focus now on the two main uncertainties that affect the predictions for the merger rates of MBHs: the initial mass function of the MBH population at high redshift; and the time delays between the merger of two DM halos and the merger of the hosted MBHs.

\section{Black-hole mass function at high redshift}

Several formation mechanisms for the initial population of black-hole seeds at high redshift have been proposed (see e.g. [80] for a review). "Light" seeds (LSs) may form from the first generation (Population III) of stars [86]. These metal-free stars are believed to form at high redshift (peaking around $z \sim 10$ ) in the deepest potential wells (i.e. in the most massive DM halos at a given epoch) and to be very massive (up to several hundred solar masses). When they explode as SNae, they may leave behind black holes of a few hundred $M_{\odot}$, which may provide the seeds for the subsequent growth (via mergers and accretion) of the MBH population. A problem with this scenario is that it is difficult to reconcile it with the AGN luminosity function at high redshift, and particularly with the discovery of active MBHs with masses $\gtrsim 10^{9} M_{\odot}$ in gas- and dust-rich galaxies at $z \gtrsim 7[51,97,6,138,137,139]$. At those redshifts the age of the universe was $\lesssim 0.77 \mathrm{Gyr}$, and it seems difficult to accumulate those huge masses in such a short time span.

In more detail, if accretion onto these early LSs is Eddington-limited, their mass grows as $M_{\mathrm{bh}} \propto e^{t / \tau}$, with the characteristic timescale $\tau$ given by

$$
\tau=\frac{\eta}{(1-\eta) \lambda} t_{\mathrm{Edd}} \approx \frac{4.5 \times 10^{7}}{\lambda} \mathrm{yr} .
$$

Here, $\lambda \equiv L / L_{\mathrm{Edd}}$ is the Eddington ratio between the black hole's bolometric luminosity and the Eddington limit $L_{\mathrm{Edd}} \approx 1.4 \times 10^{38} M_{\mathrm{bh}} / M_{\odot} \mathrm{erg} \mathrm{s}^{-1} ; t_{\mathrm{Edd}}=$ $M_{\mathrm{bh}} c^{2} / L_{\mathrm{Edd}} \approx 0.4 \mathrm{Gyr}$ is the Eddington characteristic timescale; and $\eta \equiv L /\left(\dot{M}_{\mathrm{bh}} c^{2}\right)$ is the radiative efficiency of the accretion flow, which we have set to $\eta=0.1$ (as approximately suitable for describing thin disks around slowly spinning black holes). It is therefore clear that even for $\lambda \sim 1$ (Eddington accretion) a seed of a few $\times 10^{2} M_{\odot}$ would grow to $\gtrsim 10^{9} M_{\odot}$ only at $z \sim 7$, which is marginally sufficient to explain the observations of [51, 97, 6, 138, 137, 139]. Taking into account 
that accretion is also expected to proceed intermittently (in bouts possibly associated with major mergers and/or starbursts), it seems that a LS formation scenario should require moderately super-Eddington accretion [87]. This is quite possible since at relatively high mass accretion rates $\dot{M}_{\mathrm{bh}} \gtrsim 0.5 L_{\mathrm{Edd}} / c^{2}$, accretion flows are expected to "puff up" and transition from a radiatively efficient, geometrically thin configuration to radiatively inefficient, geometrically slim disks, which can sustain moderately super-Eddington accretion [87].

Another possibility is that $\mathrm{MBH}$ seeds may form already with relatively large masses $\sim 10^{4}-10^{5} M_{\odot}$ (see [88] for a recent review). Among these "heavy seed" (HS) scenarios are e.g. the rapid formation of seeds by direct collapse of gas and dust clouds in protogalaxies, induced by mergers, bar instabilities in gaseous disks, or cold-gas inflows along filaments [142, 89, 90, 41, 42, 12]; runaway collisions (favored by mass segregation) of massive stars e.g. in metal-poor nuclear stellar clusters $[39,81,106]$ or in the high- $z$, strongly star-forming galaxies with dense gas environments that are progenitors to local early-type galaxies $[18,75]$.

\section{Delays between galaxy and black-hole mergers}

When two DM halos coalesce according to the merger-tree formalism described earlier in this Chapter, the smaller one initially retains its identity as a sub-halo (or "satellite") of the newly formed system. That sub-halo then slowly spirals in (on typical timescales of a few Gyr) as a result of dynamical friction [25]. During this phase, tidal effects (stripping and evaporation) remove mass from both the DM and baryonic components, which in turn affects the evolution of the system (making dynamical friction less and less efficient) [129].

When the sub-halo finally reaches the center of the system, the baryonic components (the "galaxies") and the contained MBHs do not coalesce immediately, but keep evolving under the same processes (dynamical friction and tidal stripping/evaporation) [9, 45, 132]. This phase, during which the two MBHs go from $\sim$ kpc to $\sim$ pc separation, can also last for several Gyr, especially when the merging galaxies have unequal stellar masses [132]. Moreover, tidal effects progressively disrupt the smaller galaxy during its evolution, eventually leaving the MBH naked or at most surrounded by a core of stars [45]. As a result, a significant number of "stalled" MBHs may be left wandering at separations of hundreds of pc [45, 132].

For the MBHs that reach separations $\sim \mathrm{pc}$ and form bound binaries, dynamical friction eventually becomes inefficient compared to other processes. Among the latter, a prominent role is played by stellar hardening [108, 119], i.e. three-body interactions between the MBH binary and individual stars. Stars on low angular momentum orbits (i.e. in the "loss cone") interact strongly with the binary, removing energy from it via the slingshot effect. Repeated interactions of this sort cause the MBH binary to shrink, while stars in the loss cone are progressively removed

from the system (being ejected e.g. as hypervelocity stars [120]). As a result, the loss cone needs to be replenished (by diffusion of stars on the stellar relaxation 
timescale) if stellar hardening is to efficiently drive the binary's evolution down to separations $\sim 10^{-2}-10^{-3} \mathrm{pc}$ (where GW emission alone can lead the system to coalescence in less than a Hubble time). Mechanisms that could help enhance stellar diffusion and thus replenish the loss cone (ensuring hardening timescales of a few Gyr) include e.g. triaxiality of the galaxy potential (resulting e.g. from a recent galaxy merger) [145, 70, 134, 135, 136] or galaxy rotation [64].

It should also be noted that in gas-rich galactic nuclei, planetary-like migration in a gaseous nuclear disk may harden the MBH binary on timescales even shorter $\left(\sim 10^{7}-10^{8} \mathrm{yr}\right)$ than those of stellar interactions [85, 34, 83, 113, 101, 47, 98]. Moreover, even in gas-poor nuclei, and even if stellar hardening becomes inefficient due to insufficient loss-cone replenishment, a third $\mathrm{MBH}$ will eventually be added to the system by a later galaxy merger (as a consequence of the hierarchical nature of structure formation, described in the first part of this Chapter) [22, 23, 9]. Triple MBH interactions can trigger the merger of the inner binary via Kozai-Lidov oscillations [74, 82], which tend to decrease the inclination of outer binary while increasing the eccentricity of the inner one, or via chaotic three-body interactions [20, 21]. Both processes can drive the inner binary to the GW-dominated regime (i.e. separations of $\sim 10^{-2}-10^{-3} \mathrm{pc}$ ) in a sizeable fraction of systems [22, 23, 9]. Remarkably, this triple-MBH merger channel leaves a characteristic imprint on the GW signal observable by LISA, since MBH binaries originating from it are expected to carry a significant residual eccentricity ( $~ 00.99$ when they enter the LISA band, and $\sim 0.1$ at coalescence) [23].

\section{Predictions for LISA and PTAs}

The predictions for how many MBH mergers will be observed by LISA, as well as for the parameters (masses, redshifts, etc) of those sources, are very sensitive to the physics outlined above, and in particular to the sub-grid modeling of the seeding mechanism, the delays between galaxy and MBH mergers, and the impact of SN feedback on MBH growth.

Table 1 Predictions of the models of [9] for the total number of MBH mergers and detections in 4 years of observation with LISA. Adapted from [9].

\begin{tabular}{|c|c|c|c|c|}
\hline \multirow{2}{*}{ Model } & \multicolumn{2}{|r|}{ LS } & \multicolumn{2}{|r|}{ HS } \\
\hline & Total & Detected & Total & Detected \\
\hline \multicolumn{5}{|c|}{ SN feedback } \\
\hline SN-Delays & 48 & 16 & 25 & 25 \\
\hline SN-shortDelays & 178 & 36 & 1269 & 1269 \\
\hline \multicolumn{5}{|c|}{ No SNfeedback } \\
\hline noSN-Delays & 192 & 146 & 10 & 10 \\
\hline noSN-shortDelays & 1159 & 307 & 1288 & 1288 \\
\hline
\end{tabular}




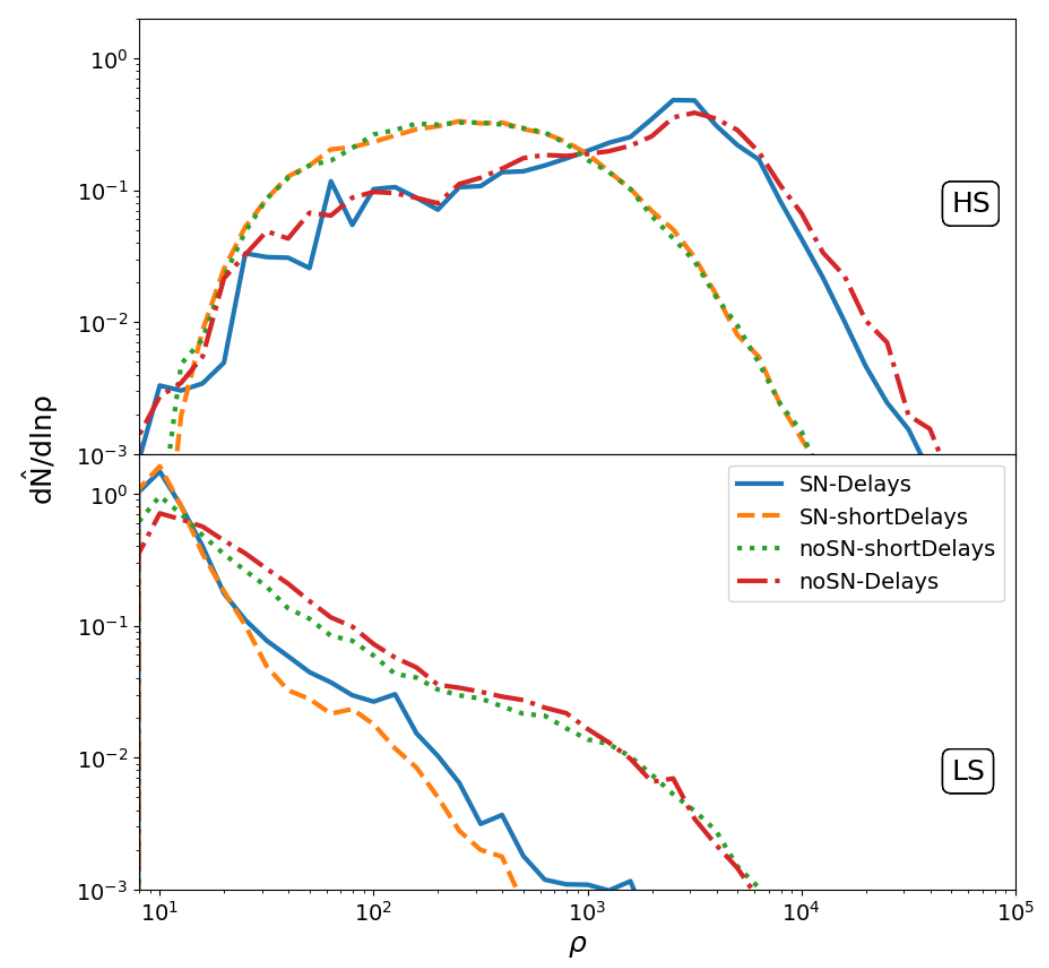

Fig. 8 Distribution of signal-to-noise ratio (normalized to the total number of detections) for the HS and LS models (respectively upper and lower panels). Adapted from [9].

To illustrate this fact, we will review here the results of the semi-analytic galaxyformation model of [9] (based in turn on the model of [7], with the incremental improvements described in [121, 1, 22, 23] and in [9] itself). We will focus in particular on two competing scenarios for the black-hole seeds, namely a population-III LS scenario [86], and a representative HS model [142] where seeds form (with masses $\sim 10^{4}-\sim 10^{5} M_{\odot}$ ) by direct collapse, as result of bar instabilities in high-redshift protogalaxies. Moreover, besides considering versions of both the LS and HS models where SN feedback on the nuclear gas and delays are included (SN-Delays models), we also review results obtained when either (or both) of these physical processes are switched off or modified. In particular, in the noSN models we neglect the effect of SN explosions on the nuclear gas. In the shortDelays models, we switch off the delays occurring as the MBH pair moves from $\mathrm{kpc}$ to pc separations (i.e. we neglect dynamical friction on the satellite galaxy and/or its $\mathrm{MBH}$ ), while keeping the delays due to dynamical friction of the DM halos, as well as those due to stellar hardening, gas-driven migration, and triple MBH interactions. (Note that the shortDelays models correspond to the nodelays models of [9], since in that work the "delays" 


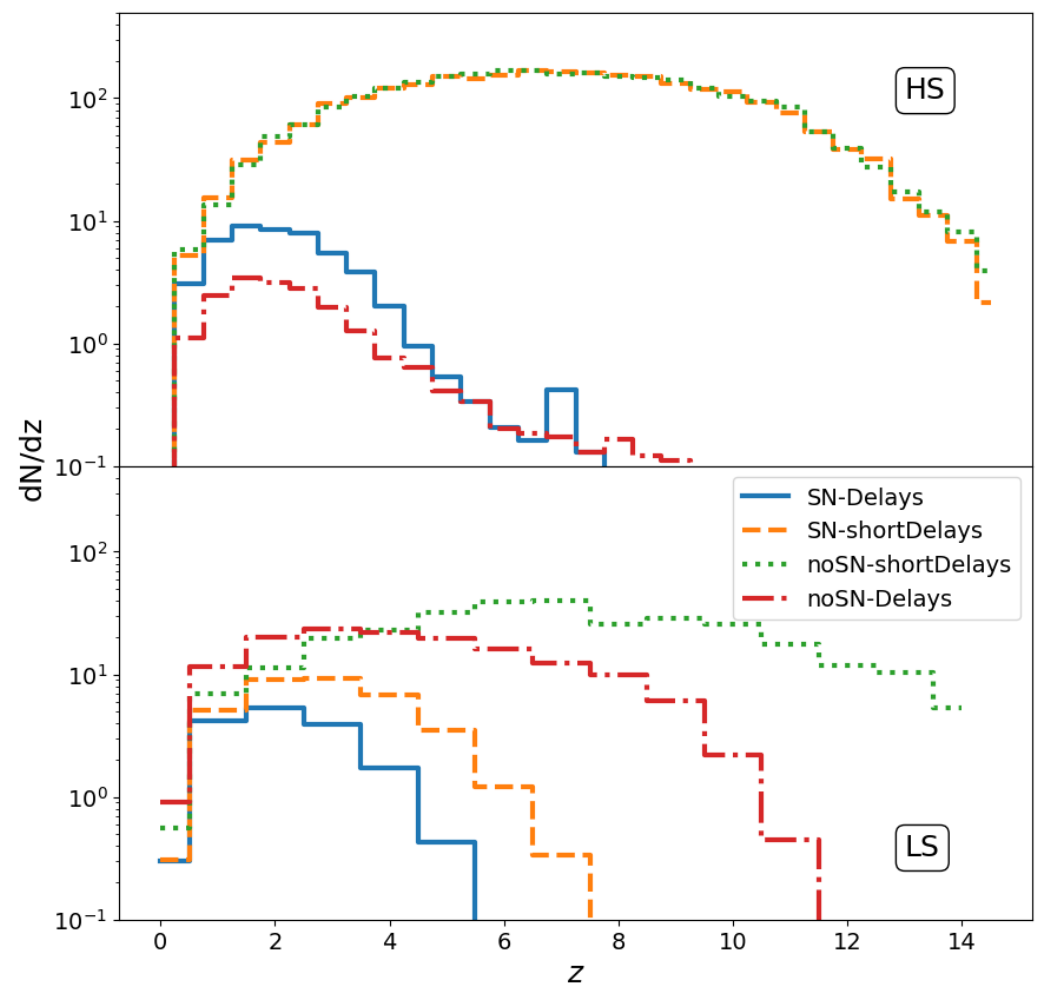

Fig. 9 Detected MBH mergers per unit redshift in 4 years of LISA observations, for the HS models (upper panel) and the LS models (lower panel). Adapted from [9].

are only meant as those due to dynamical friction on the satellite galaxy and/or its MBH.)

The (average) number of MBH mergers detectable by LISA in 4 years of observation is reported in Table 1, alongside the total number of events (i.e. the number of mergers that could be detected in the same observation time if the detector had infinite sensitivity). We assume a signal-to-noise ratio $(\rho)$ detection threshold $\rho>8$. As can be seen, LISA will detect essentially all MBH coalescences in our past light cone in the HS models. That is the result of their large masses, which produce larger signal-to-noise ratios (cf. also [71]). Conversely, in the LS models, the fraction of detected MBH mergers is always less than one because of the lower binary masses, which translate into lower signal-to-noise ratios and GW frequencies at the high-end of the LISA sensitivity curve. The detection fraction is typically around $20-30 \%$, but can reach $\sim 75 \%$ in the noSN-Delays (where seed growth is unhampered and the delays allow for longer MBH growth before binaries merge, yielding higher masses and signal-to-noise ratios). Note also that the inclusion of realistic delays tends to decrease both the intrinsic number of MBH mergers and the number of detections. This effect is more spectacular in the case of HSs. 


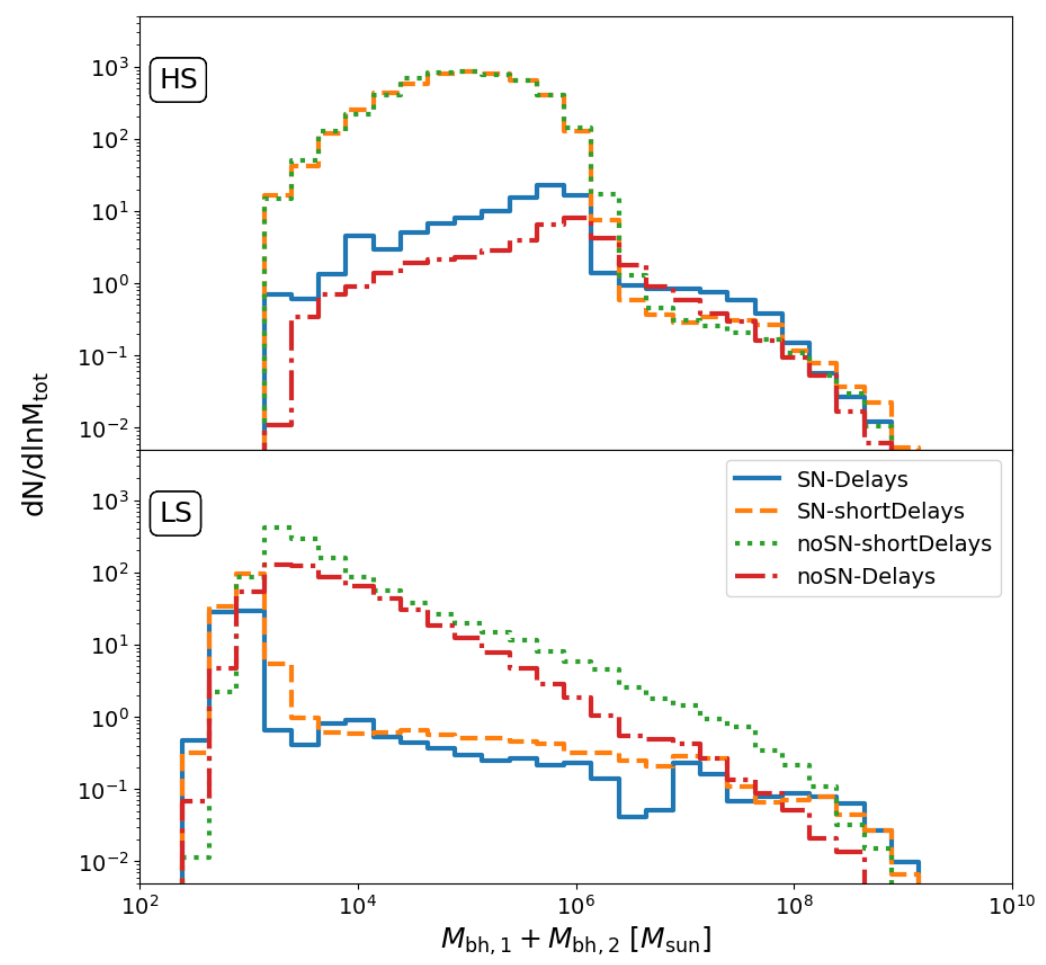

Fig. 10 Detected MBH mergers as a function of total MBH binary mass for the HS models (upper panel) and the LS models (lower panel). Adapted from [9].

The distribution of the signal-to-noise ratios of the detected mergers is shown in Fig. 8. As expected, mergers from HS models are typically louder than those from LS models, as a result of their larger masses. One can also see that the differences between the HS models are mainly due to the dynamics, with realistic delay timescales resulting in louder sources (because longer delays allow the MBHs to grow to larger masses, and shift mergers to lower redshifts, where signal-to-noise ratios are naturally higher). In the LS models, the spread in the predictions is instead due mainly to SN feedback, which lowers the typical mass of MBH binaries by hampering their early growth. Note that the relatively low signal-to-noise ratios of the LS models highlight the importance of the LISA sensitivity level at high frequencies, where these light sources are detected.

The distribution of the detected mergers in redshift and in total mass of the MBH binary is shown in Figs. 9-10. Again, the effect of the delays, which decrease the number of observed systems and shift the event distribution to lower redshift, is particularly evident. As for SN feedback, it mainly affects LS models. Since it tends to prevent the MBHs from growing when they are located in shallow potential wells at high redshift, the redshift distribution peaks later in the LS models with SN feedback. Those LS models also present a noticeable suppression in the number of merg- 


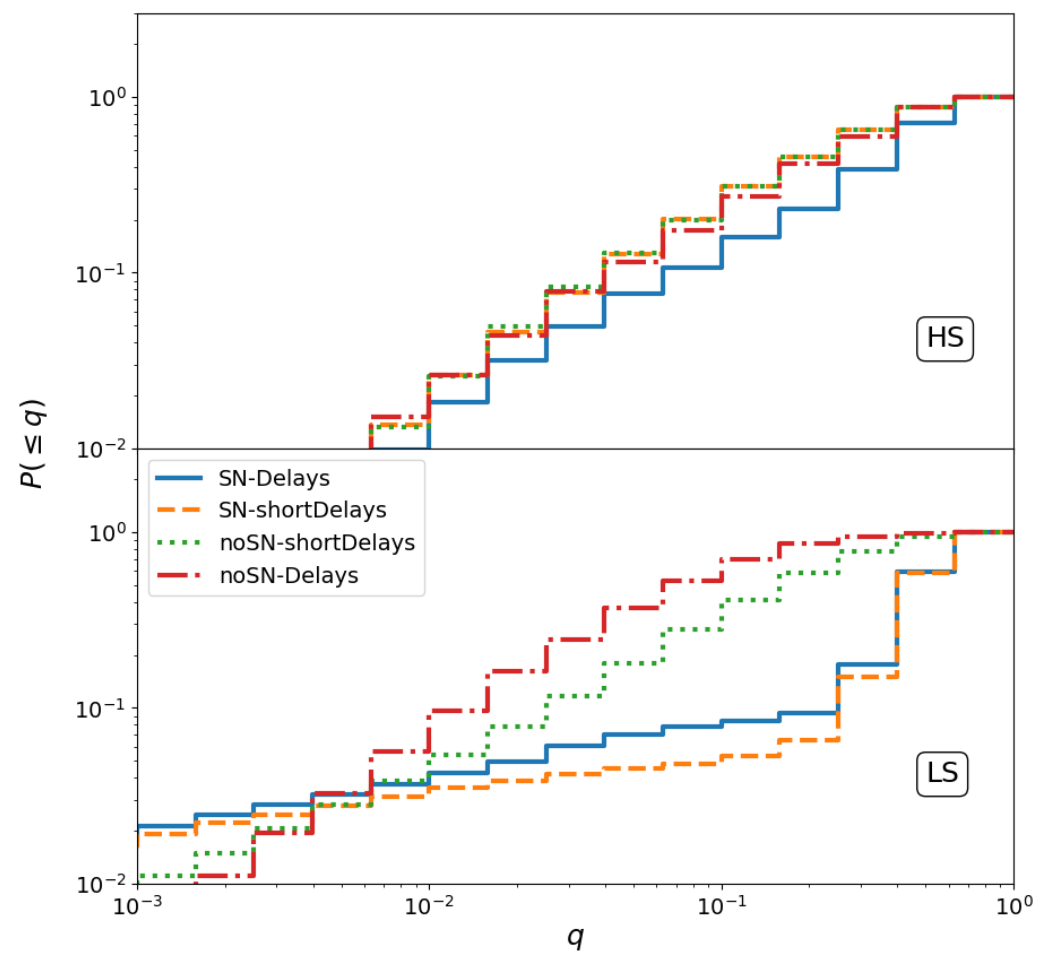

Fig. 11 Fraction of detected MBH mergers with mass ratio below $q$, for the HS models (upper panel) and the LS models (lower panel). Adapted from [9].

ers with masses below $\sim 10^{7} M_{\odot}$, which is responsible for the lower number of detections in Table 1. Also note that the low-mass peak in the distribution of the HS binaries in the models with short delays occurs because the MBH seeds do not have time to grow significantly before merging.

Particularly important for modeling GW signals is the mass ratio of MBH binaries, whose distribution is shown in Fig. 11. As can be seen, most systems feature mass ratios (defined as $q=M_{\mathrm{bh}, 2} / M_{\mathrm{bh}, 1} \leq 1$ ) above $0.01-0.1$, according to the specific model. Interestingly, the predicted distribution is very robust in HS models, while in LS ones SN feedback tends to favor comparable-mass systems. This can be understood by noting that LS models with no feedback allow seeds to grow more quickly away from their initial masses, producing a larger range of possible mass ratios. When the seed growth is impeded by SN feedback, however, a significant number of mergers take place between black holes with masses close to their initial values, which are similar as they are produced by the same physical mechanism (explosions of population-III stars).

Finally, let us comment that while the number of sources detected by LISA, as well as their parameters, is to a large extent model dependent, the predictions for the level of the stochastic background in the frequency band of PTAs are instead quite 


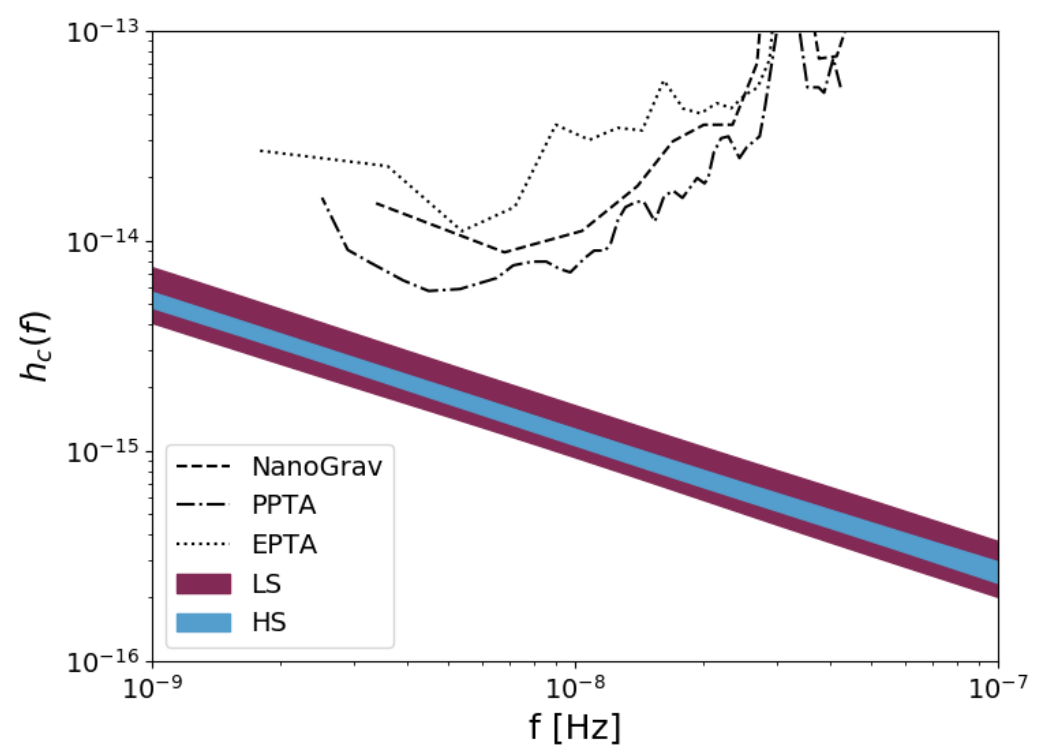

Fig. 12 Characteristic strain of the stochastic background from MBH binaries in the PTA band, for the eight models considered in this Chapter (with the envelope of the LS/HS model predictions shown in purple/light blue, respectively). Also shown are the sensitivities of ongoing PTA experiments [3, 124, 38]. Adapted from [9].

robust to the assumptions made about the seeding mechanism, the delays between galaxy and MBH mergers, and the effect of SN feedback on nuclear gas. This is evident from Fig. 12, which shows the predictions for the characteristic strain $h_{c}$ of the stochastic background from MBH mergers in the $\sim \mathrm{nHz}$ frequency band, for the eight models presented above, compared with the upper bounds from EPTA, PPTA and NANOGrav [3, 124, 38]. Note that the slope of the predictions $\left(h_{c} \propto f^{-2 / 3}\right)$ simply follows from the quadrupole formula of general relativity, while the normalization (which depends on the MBH population) only shows a minor scatter according to the model. This robust dependence on the astrophysical model is expected (cf. for instance also [22]), because the PTA signal comes from comparable-mass MBH binaries with masses $\gtrsim 10^{8} M_{\odot}$ at $z \lesssim 2$. The predictions for such systems depend very weakly on the initial seed mass function (since memory of the latter is lost due to accretion) and on the modeling of the delays between galaxy and $\mathrm{MBH}$ mergers (which for these systems are typically short). One physical ingredient that may affect the normalization of the PTA signal, however, is the overall normalization of the black hole-galaxy scaling relations [122]. The semi-analytic models presented in this Chapter were calibrated to the observed scaling relations, accounting for the selection bias affecting the latter [8]. In particular, as shown by [123], the black-hole sphere of influence must be resolved for the MBH mass to be reliably estimated, which may bias the inferred normalization of the black hole-galaxy scaling relations. 


\section{Future prospects}

While this Chapter was being finalized, NANOGrav reported evidence for a commonspectrum low-frequency stochastic process that affects pulsar-timing residuals in their 12.5-year dataset [4]. The origin of this common "red noise" may be a stochastic GW background from MBH mergers. NANOgrav has so far reported no convincing evidence of quadrupolar angular correlations among the residuals of their pulsars (i.e. the Hellings-Downs correlation that should be present for a GW background [63]). However, if one interprets this common "red noise" as tentatively due to GWs, that would correspond to $h_{c}=A(f \mathrm{yr})^{-2 / 3}$, with $A=1.92 \times 10^{-15}$ (median) and $5 \%-95 \%$ quantiles $A=1.37-2.67 \times 10^{-15}$. This would be above the previously published upper bounds shown in Fig. 12, although NANOgrav attributes this discrepancy to the (optimistic) use of log-flat priors in earlier analyses (including theirs). While it remains to be ascertained whether NANOgrav has really observed the stochastic background from MBH binaries, it is possible that the predictions of this Chapter for LISA may need to be recalibrated (perhaps reconsidering the effect of selection effects on the scaling relations) and thus possibly revised to higher event rates. In any case, even the models shown in Fig. 12 would be testable by PTAs with $\approx 15-20 \mathrm{yr}$ of residual collection (assuming about $50 \mathrm{~ms}$ pulsars monitored at $100 \mathrm{~ns}$ precision). A much earlier detection would be possible with the Square Kilometer Array (SKA) telescope [48].

\section{Acknowledgments}

E.B. acknowledges financial support provided under the European Union's H2020 ERC Consolidator Grant "GRavity from Astrophysical to Microscopic Scales" grant agreement no. GRAMS-815673. E.B. thanks I. Dvorkin, M. Bonetti, M. Tremmel and M. Volonteri for numerous insightful conversations on the astrophysics of galaxies and black holes, and for agreeing to adapting the figures of [9]. A.L. acknowledges financial support from the EU H2020-MSCAITN-2019 Project 860744 'BiD4BEST: Big Data applications for Black hole Evolution STudies' and from the PRIN MIUR 2017 prot. 20173ML3WW 002, 'Opening the ALMA window on the cosmic evolution of gas, stars and massive black holes'. We thank J. Gonzalez for proof-reading this manuscript.

\section{References}

[1] Antonini F, Barausse E, Silk J (2015) The Coevolution of Nuclear Star Clusters, Massive Black Holes, and Their Host Galaxies. The Astrophysical Journal 812:72 
[2] Antonini F, Barausse E, Silk J (2015) The Imprint of Massive Black Hole Mergers on the Correlation between Nuclear Star Clusters and Their Host Galaxies. The Astrophysical Journal Letters 806:L8

[3] Arzoumanian Z, Baker PT, Brazier A, Burke- Spolaor S, Chamberlin SJ, Chatterjee S, Christy B, Cordes JM, Cornish NJ, Crawford F, Thankful Cromartie H, Crowter K, DeCesar M, Demorest PB, Dolch T, Ellis JA, Ferdman RD, Ferrara E, Folkner WM, Fonseca E, Garver-Daniels N, Gentile PA, Haas R, Hazboun JS, Huerta EA, Islo K, Jones G, Jones ML, Kaplan DL, Kaspi VM, Lam MT, Lazio TJW, Levin L, Lommen AN, Lorimer DR, Luo J, Lynch RS, Madison DR, McLaughlin MA, McWilliams ST, Mingarelli CMF, Ng C, Nice DJ, Park RS, Pennucci TT, Pol NS, Ransom SM, Ray PS, Rasskazov A, Siemens X, Simon J, Spiewak R, Stairs IH, Stinebring DR, Stovall K, Swiggum J, Taylor SR, Vallisneri M, van Haasteren R, Vigeland S, Zhu WW, NANOGrav Collaboration (2018) The NANOGrav 11 Year Data Set: Pulsar-timing Constraints on the Stochastic Gravitational-wave Background. The Astrophysical Journal 859:47

[4] Arzoumanian Z, Baker PT, Blumer H, Becsy B, Brazier A, Brook PR, BurkeSpolaor S, Chatterjee S, Chen S, Cordes JM, Cornish NJ, Crawford F, Cromartie HT, DeCesar ME, Demorest PB, Dolch T, Ellis JA, Ferrara EC, Fiore W, Fonseca E, Garver-Daniels N, Gentile PA, Good DC, Hazboun JS, Holgado AM, Islo K, Jennings RJ, Jones ML, Kaiser AR, Kaplan DL, Kelley LZ, Key JS, Laal N, Lam MT, Lazio TJW, Lorimer DR, Luo J, Lynch RS, Madison DR, McLaughlin MA, Mingarelli CMF, Ng C, Nice DJ, Pennucci TT, Pol NS, Ransom SM, Ray PS, Shapiro-Albert BJ, Siemens X, Simon J, Spiewak R, Stairs IH, Stinebring DR, Stovall K, Sun JP, Swiggum JK, Taylor SR, Turner JE, Vallisneri M, Vigeland SJ, Witt CA (2020) The nanograv 12.5-year data set: Search for an isotropic stochastic gravitational-wave background. in press

[5] Baldassare VF, Geha M, Greene J (2019) A search for optical AGN variability in 35,000 low-mass galaxies with the Palomar Transient Factory. arXiv e-prints arXiv: 1910.06342

[6] Bañados E, Carilli C, Walter F, Momjian E, Decarli R, Farina EP, Mazzucchelli C, Venemans BP (2018) A powerful radio-loud quasar at the end of cosmic reionization. The Astrophysical Journal 861(2):L14

[7] Barausse E (2012) The evolution of massive black holes and their spins in their galactic hosts. Monthly Notices of the Royal Astronomical Society 423:2533-2557

[8] Barausse E, Shankar F, Bernardi M, Dubois Y, Sheth RK (2017) Selection bias in dynamically measured supermassive black hole samples: scaling relations and correlations between residuals in semi-analytic galaxy formation models. Monthly Notices of the Royal Astronomical Society 468:4782-4791

[9] Barausse E, Dvorkin I, Tremmel M, Volonteri M, Bonetti M (2020) Massive black hole merger rates: the effect of kpc separation wandering and supernova feedback. arXiv e-prints arXiv:2006.03065 
[10] Bardeen JM, Bond JR, Kaiser N, Szalay AS (1986) The Statistics of Peaks of Gaussian Random Fields. The Astrophysical Journal 304:15

[11] Begelman MC, Blandford RD, Rees MJ (1980) Massive black hole binaries in active galactic nuclei. Nature 287:307-309

[12] Begelman MC, Rossi EM, Armitage PJ (2008) Quasi-stars: accreting black holes inside massive envelopes. Monthly Notices of the Royal Astronomical Society 387(4):1649-1659

[13] Behroozi PS, Wechsler RH, Conroy C (2013) The Average Star Formation Histories of Galaxies in Dark Matter Halos from $\mathrm{z}=0-8$. The Astrophysical Journal 770(1):57

[14] Bekki K, Graham AW (2010) On the Transition from Nuclear-cluster- to Black-hole-dominated Galaxy Cores. ApJL 714(2):L313-L317

[15] Bhattacharya S, Heitmann K, White M, Lukić Z, Wagner C, Habib S (2011) Mass Function Predictions Beyond $\Lambda$ CDM. The Astrophysical Journal 732(2): 122

[16] Blandford RD, Payne DG (1982) Hydromagnetic flows from accretion disks and the production of radio jets. Monthly Notices of the Royal Astronomical Society 199:883-903

[17] Blandford RD, Znajek RL (1977) Electromagnetic extraction of energy from Kerr black holes. Monthly Notices of the Royal Astronomical Society 179:433-456

[18] Boco L, Lapi A, Danese L (2020) Growth of Supermassive Black Hole Seeds in ETG Star-forming Progenitors: Multiple Merging of Stellar Compact Remnants via Gaseous Dynamical Friction and Gravitational-wave Emission. The Astrophysical Journal 891(1):94

[19] Bond JR, Cole S, Efstathiou G, Kaiser N (1991) Excursion Set Mass Functions for Hierarchical Gaussian Fluctuations. The Astrophysical Journal 379:440

[20] Bonetti M, Haardt F, Sesana A, Barausse E (2016) Post-Newtonian evolution of massive black hole triplets in galactic nuclei - I. Numerical implementation and tests. Monthly Notices of the Royal Astronomical Society 461:4419-4434

[21] Bonetti M, Haardt F, Sesana A, Barausse E (2018) Post-Newtonian evolution of massive black hole triplets in galactic nuclei - II. Survey of the parameter space. Monthly Notices of the Royal Astronomical Society 477:3910-3926

[22] Bonetti M, Sesana A, Barausse E, Haardt F (2018) Post-Newtonian evolution of massive black hole triplets in galactic nuclei - III. A robust lower limit to the $\mathrm{nHz}$ stochastic background of gravitational waves. Monthly Notices of the Royal Astronomical Society 477:2599-2612

[23] Bonetti M, Sesana A, Haardt F, Barausse E, Colpi M (2019) Post-Newtonian evolution of massive black hole triplets in galactic nuclei - IV. Implications for LISA. Monthly Notices of the Royal Astronomical Society 486(3):40444060

[24] Bower RG, Benson AJ, Malbon R, Helly JC, Frenk CS, Baugh CM, Cole S, Lacey CG (2006) Breaking the hierarchy of galaxy formation. Monthly Notices of the Royal Astronomical Society 370(2):645-655 
[25] Boylan-Kolchin M, Ma CP, Quataert E (2008) Dynamical friction and galaxy merging time-scales. Monthly Notices of the Royal Astronomical Society 383:93-101

[26] Bryan GL, Norman ML (1998) Statistical Properties of X-Ray Clusters: Analytic and Numerical Comparisons. The Astrophysical Journal 495(1):80-99

[27] Bullock JS, Dekel A, Kolatt TS, Kravtsov AV, Klypin AA, Porciani C, Primack JR (2001) A Universal Angular Momentum Profile for Galactic Halos. The Astrophysical Journal 555(1):240-257

[28] Cattaneo A, Dekel A, Devriendt J, Guiderdoni B, Blaizot J (2006) Modelling the galaxy bimodality: shutdown above a critical halo mass. Monthly Notices of the Royal Astronomical Society 370:1651-1665

[29] Cimatti A, Fraternali F, Nipoti C (2019) Introduction to Galaxy Formation and Evolution. Cambridge Univ. Press, Cambridge

[30] Cole S, Aragon-Salamanca A, Frenk CS, Navarro JF, Zepf SE (1994) A recipe for galaxy formation. Monthly Notices of the Royal Astronomical Society 271:781-806

[31] Cole S, Lacey CG, Baugh CM, Frenk CS (2000) Hierarchical galaxy formation. Monthly Notices of the Royal Astronomical Society 319(1):168-204

[32] Correa CA, Schaye J, Wyithe JSB, Duffy AR, Theuns T, Crain RA, Bower RG (2018) The formation of hot gaseous haloes around galaxies. Monthly Notices of the Royal Astronomical Society 473(1):538-559

[33] Croton DJ, Springel V, White SDM, De Lucia G, Frenk CS, Gao L, Jenkins A, Kauffmann G, Navarro JF, Yoshida N (2006) The many lives of active galactic nuclei: cooling flows, black holes and the luminosities and colours of galaxies. Monthly Notices of the Royal Astronomical Society 365:11-28

[34] Cuadra J, Armitage PJ, Alexander RD, Begelman MC (2009) Massive black hole binary mergers within subparsec scale gas discs. Monthly Notices of the Royal Astronomical Society 393:1423-1432

[35] Daddi E, Elbaz D, Walter F, Bournaud F, Salmi F, Carilli C, Dannerbauer H, Dickinson M, Monaco P, Riechers D (2010) Different Star Formation Laws for Disks Versus Starbursts at Low and High Redshifts. The Astrophysical Journal Letters 714(1):L118-L122

[36] Dekel A, Birnboim Y (2006) Galaxy bimodality due to cold flows and shock heating. Monthly Notices of the Royal Astronomical Society 368:2-20

[37] Dekel A, Birnboim Y, Engel G, Freundlich J, Goerdt T, Mumcuoglu M, Neistein E, Pichon C, Teyssier R, Zinger E (2009) Cold streams in early massive hot haloes as the main mode of galaxy formation. Nature 457:451-454

[38] Desvignes G, Caballero RN, Lentati L, Verbiest JPW, Champion DJ, Stappers BW, Janssen GH, Lazarus P, Osłowski S, Babak S, Bassa CG, Brem P, Burgay M, Cognard I, Gair JR, Graikou E, Guillemot L, Hessels JWT, Jessner A, Jordan C, Karuppusamy R, Kramer M, Lassus A, Lazaridis K, Lee KJ, Liu K, Lyne AG, McKee J, Mingarelli CMF, Perrodin D, Petiteau A, Possenti A, Purver MB, Rosado PA, Sanidas S, Sesana A, Shaifullah G, Smits R, Taylor SR, Theureau G, Tiburzi C, van Haasteren R, Vecchio A (2016) High- 
precision timing of 42 millisecond pulsars with the European Pulsar Timing Array. Monthly Notices of the Royal Astronomical Society 458:3341-3380

[39] Devecchi B, Volonteri M, Rossi EM, Colpi M, Portegies Zwart S (2012) High-redshift formation and evolution of central massive objects - II. The census of BH seeds. Monthly Notices of the Royal Astronomical Society 421(2):1465-1475

[40] Di Matteo T, Springel V, Hernquist L (2005) Energy input from quasars regulates the growth and activity of black holes and their host galaxies. Nature 433:604-607

[41] Di Matteo T, Khandai N, DeGraf C, Feng Y, Croft RAC, Lopez J, Springel V (2012) Cold Flows and the First Quasars. The Astrophysical Journal Letters 745(2):L29

[42] Di Matteo T, Croft RAC, Feng Y, Waters D, Wilkins S (2017) The origin of the most massive black holes at high-z: BlueTides and the next quasar frontier. Monthly Notices of the Royal Astronomical Society 467(4):42434251

[43] Diemand J, Moore B, Stadel J (2005) Earth-mass dark-matter haloes as the first structures in the early Universe. Nature 433(7024):389-391

[44] Diemer B, Mansfield P, Kravtsov AV, More S (2017) The Splashback Radius of Halos from Particle Dynamics. II. Dependence on Mass, Accretion Rate, Redshift, and Cosmology. The Astrophysical Journal 843(2):140

[45] Dosopoulou F, Antonini F (2017) Dynamical Friction and the Evolution of Supermassive Black Hole Binaries: The Final Hundred-parsec Problem. The Astrophysical Journal 840:31

[46] Dubois Y, Devriendt J, Slyz A, Teyssier R (2012) Self-regulated growth of supermassive black holes by a dual jet-heating active galactic nucleus feedback mechanism: methods, tests and implications for cosmological simulations. Monthly Notices of the Royal Astronomical Society 420(3):2662-2683

[47] Duffell PC, D’Orazio D, Derdzinski A, Haiman Z, MacFadyen A, Rosen AL, Zrake J (2019) Circumbinary Disks: Accretion and Torque as a Function of Mass Ratio and Disk Viscosity. arXiv e-prints arXiv:1911.05506

[48] Dvorkin I, Barausse E (2017) The nightmare scenario: measuring the stochastic gravitational wave background from stalling massive black hole binaries with pulsar timing arrays. Monthly Notices of the Royal Astronomical Society 470(4):4547-4556

[49] Eke VR, Cole S, Frenk CS (1996) Cluster evolution as a diagnostic for Omega. Monthly Notices of the Royal Astronomical Society 282:263-280

[50] Fakhouri O, Ma CP, Boylan-Kolchin M (2010) The merger rates and mass assembly histories of dark matter haloes in the two Millennium simulations. Monthly Notices of the Royal Astronomical Society 406(4):2267-2278

[51] Fan X, Strauss MA, Richards GT, Hennawi JF, Becker RH, White RL, Diamond-Stanic AM, Donley JL, Jiang L, Kim JS, Vestergaard M, Young JE, Gunn JE, Lupton RH, Knapp GR, Schneider DP, Brandt WN, Bahcall NA, Barentine JC, Brinkmann J, Brewington HJ, Fukugita M, Harvanek M, Kleinman SJ, Krzesinski J, Long D, Neilsen J Eric H, Nitta A, Snedden SA, 
Voges W (2006) A Survey of z⿺5.7 Quasars in the Sloan Digital Sky Survey. IV. Discovery of Seven Additional Quasars. The Astronomical Journal 131(3):1203-1209

[52] Ferrarese L, Côté P, Dalla Bontà E, Peng EW, Merritt D, Jordán A, Blakeslee JP, Hașegan M, Mei S, Piatek S, Tonry JL, West MJ (2006) A Fundamental Relation between Compact Stellar Nuclei, Supermassive Black Holes, and Their Host Galaxies. The Astrophysical Journal Letters 644(1):L21-L24

[53] Frank J, King A, Raine D (2002) Accretion Power in Astrophysics. Cambridge University Press

[54] Fujita A, Mac Low MM, Ferrara A, Meiksin A (2004) Cosmological Feedback from High-Redshift Dwarf Galaxies. The Astrophysical Journal 613(1):159-179

[55] Gehren T, Fried J, Wehinger PA, Wyckoff S (1984) Host galaxies of quasars and their association with galaxy clusters. The Astrophysical Journal 278:1127

[56] Genel S, Bouché N, Naab T, Sternberg A, Genzel R (2010) The Growth of Dark Matter Halos: Evidence for Significant Smooth Accretion. The Astrophysical Journal 719(1):229-239

[57] Genzel R, Tacconi LJ, Gracia-Carpio J, Sternberg A, Cooper MC, Shapiro K, Bolatto A, Bouché N, Bournaud F, Burkert A, Combes F, Comerford J, Cox P, Davis M, Schreiber NMF, Garcia-Burillo S, Lutz D, Naab T, Neri R, Omont A, Shapley A, Weiner B (2010) A study of the gas-star formation relation over cosmic time. Monthly Notices of the Royal Astronomical Society 407(4):2091-2108

[58] Giocoli C, Tormen G, Sheth RK (2012) Formation times, mass growth histories and concentrations of dark matter haloes. Monthly Notices of the Royal Astronomical Society 422(1):185-198

[59] Graham AW (2016) Black hole and nuclear cluster scaling relations: $\mathrm{M}_{b h}$ $\sim \mathrm{M}_{n c}{ }^{2.7+/-0.7}$. In: Meiron Y, Li S, Liu FK, Spurzem R (eds) Star Clusters and Black Holes in Galaxies across Cosmic Time, vol 312, pp 269-273, DOI 10.1017/S1743921315008017, 1412.5715

[60] Graham AW, Spitler LR (2009) Quantifying the coexistence of massive black holes and dense nuclear star clusters. MNRAS 397(4):2148-2162

[61] Greene JE, Strader J, Ho LC (2019) Intermediate-Mass Black Holes. arXiv e-prints arXiv: 1911.09678

[62] Habouzit M, Volonteri M, Dubois Y (2017) Blossoms from black hole seeds: properties and early growth regulated by supernova feedback. Monthly Notices of the Royal Astronomical Society 468(4):3935-3948

[63] Hellings RW, Downs GS (1983) Upper limits on the isotropic gravitational radiation background from pulsar timing analysis. The Astrophysical Journal Letters 265:L39-L42

[64] Holley-Bockelmann K, Khan FM (2015) Galaxy Rotation and Rapid Supermassive Binary Coalescence. The Astrophysical Journal 810:139

[65] Hopkins PF, Cox TJ, Kereš D, Hernquist L (2008) A Cosmological Framework for the Co-Evolution of Quasars, Supermassive Black Holes, and Ellip- 
tical Galaxies. II. Formation of Red Ellipticals. The Astrophysical Journals 175(2):390-422

[66] Jenkins A, Frenk CS, White SDM, Colberg JM, Cole S, Evrard AE, Couchman HMP, Yoshida N (2001) The mass function of dark matter haloes. Monthly Notices of the Royal Astronomical Society 321(2):372-384

[67] Jiang F, van den Bosch FC (2014) Generating merger trees for dark matter haloes: a comparison of methods. Monthly Notices of the Royal Astronomical Society 440(1):193-207

[68] Kauffmann G, White SDM (1993) The merging history of dark matter haloes in a hierarchical universe. Monthly Notices of the Royal Astronomical Society 261:921-928

[69] Kauffmann G, White SDM, Guiderdoni B (1993) The formation and evolution of galaxies within merging dark matter haloes. Monthly Notices of the Royal Astronomical Society 264:201-218

[70] Khan FM, Just A, Merritt D (2011) Efficient Merger of Binary Supermassive Black Holes in Merging Galaxies. The Astrophysical Journal 732(2):89

[71] Klein A, Barausse E, Sesana A, Petiteau A, Berti E, Babak S, Gair J, Aoudia S, Hinder I, Ohme F, Wardell B (2016) Science with the space-based interferometer eLISA: Supermassive black hole binaries. Physical Review D 93(2):024003

[72] Kormendy J, Ho LC (2013) Coevolution (Or Not) of Supermassive Black Holes and Host Galaxies. Annual Review of Astronomy and Astrophysics 51:511-653

[73] Kormendy J, Richstone D (1995) Inward Bound-The Search For Supermassive Black Holes In Galactic Nuclei. Annual Review of Astronomy and Astrophysics 33:581

[74] Kozai Y (1962) Secular perturbations of asteroids with high inclination and eccentricity. The Astronomical Journal 67:591

[75] Kroupa P, Subr L, Jerabkova T, Wang L (2020) Very high redshift quasars and the rapid emergence of supermassive black holes. Monthly Notices of the Royal Astronomical Society 498(4):5652-5683

[76] Lacey C, Cole S (1993) Merger rates in hierarchical models of galaxy formation. Monthly Notices of the Royal Astronomical Society 262(3):627-649

[77] Lapi A, Cavaliere A (2011) Self-similar Dynamical Relaxation of Dark Matter Halos in an Expanding Universe. The Astrophysical Journal 743(2):127

[78] Lapi A, Danese L (2020) A Stochastic Theory of the Hierarchical Clustering I. Halo Mass Function. arXiv e-prints arXiv:2009.07023

[79] Lapi A, Salucci P, Danese L (2013) Statistics of Dark Matter Halos from the Excursion Set Approach. The Astrophysical Journal 772(2):85

[80] Latif MA, Ferrara A (2016) Formation of supermassive black hole seeds. Publ Astron Soc Austral 33:e051

[81] Latif MA, Ferrara A (2016) Formation of Supermassive Black Hole Seeds. Publications of the Astronomical Society of Australia 33:e051 
[82] Lidov ML (1962) The evolution of orbits of artificial satellites of planets under the action of gravitational perturbations of external bodies. Planetary and Space Science 9:719-759

[83] Lodato G, Nayakshin S, King AR, Pringle JE (2009) Black hole mergers: can gas discs solve the 'final parsec' problem? Monthly Notices of the Royal Astronomical Society 398:1392-1402

[84] Macciò AV, Dutton AA, van den Bosch FC (2008) Concentration, spin and shape of dark matter haloes as a function of the cosmological model: WMAP1, WMAP3 and WMAP5 results. Monthly Notices of the Royal Astronomical Society 391(4):1940-1954

[85] MacFadyen AI, Milosavljević M (2008) An Eccentric Circumbinary Accretion Disk and the Detection of Binary Massive Black Holes. The Astrophysical Journal 672:83-93

[86] Madau P, Rees MJ (2001) Massive Black Holes as Population III Remnants. The Astrophysical Journal Letters 551:L27-L30

[87] Madau P, Haardt F, Dotti M (2014) Super-critical Growth of Massive Black Holes from Stellar-mass Seeds. The Astrophysical Journal Letters 784:L38

[88] Mayer L, Bonoli S (2019) The route to massive black hole formation via merger-driven direct collapse: a review. Reports on Progress in Physics 82(1):016901

[89] Mayer L, Kazantzidis S, Escala A, Callegari S (2010) Direct formation of supermassive black holes via multi-scale gas inflows in galaxy mergers. Nature 466(7310):1082-1084

[90] Mayer L, Fiacconi D, Bonoli S, Quinn T, Roškar R, Shen S, Wadsley J (2015) Direct Formation of Supermassive Black Holes in Metal-enriched Gas at the Heart of High-redshift Galaxy Mergers. The Astrophysical Journal 810(1):51

[91] McConnell NJ, Ma CP (2013) Revisiting the Scaling Relations of Black Hole Masses and Host Galaxy Properties. The Astrophysical Journal 764:184

[92] Mo H, van den Bosch F, White S (2009) Galaxy Formation and Evolution. Cambridge University Press

[93] Mo HJ, White SDM (1996) An analytic model for the spatial clustering of dark matter haloes. Monthly Notices of the Royal Astronomical Society 282(2):347-361

[94] Mo HJ, Mao S, White SDM (1998) The formation of galactic discs. Monthly Notices of the Royal Astronomical Society 295(2):319-336

[95] Monaco P, Fontanot F, Taffoni G (2007) The MORGANA model for the rise of galaxies and active nuclei. Monthly Notices of the Royal Astronomical Society 375(4):1189-1219

[96] More S, Diemer B, Kravtsov AV (2015) The Splashback Radius as a Physical Halo Boundary and the Growth of Halo Mass. The Astrophysical Journal 810(1):36

[97] Mortlock DJ, Warren SJ, Venemans BP, Patel M, Hewett PC, McMahon RG, Simpson C, Theuns T, Gonzáles-Solares EA, Adamson A, Dye S, Hambly NC, Hirst P, Irwin MJ, Kuiper E, Lawrence A, Röttgering HJA (2011) A luminous quasar at a redshift of $\mathrm{z}=7.085$. Nature 474(7353):616-619 
[98] Muñoz DJ, Miranda R, Lai D (2019) Hydrodynamics of Circumbinary Accretion: Angular Momentum Transfer and Binary Orbital Evolution. The Astrophysical Journal 871(1):84

[99] Navarro JF, Frenk CS, White SDM (1997) A Universal Density Profile from Hierarchical Clustering. The Astrophysical Journal 490(2):493-508

[100] Nelson D, Pillepich A, Springel V, Pakmor R, Weinberger R, Genel S, Torrey P, Vogelsberger M, Marinacci F, Hernquist L (2019) First Results from the TNG50 Simulation: Galactic outflows driven by supernovae and black hole feedback. Monthly Notices of the Royal Astronomical Society p 2010

[101] Nixon CJ, Cossins PJ, King AR, Pringle JE (2011) Retrograde accretion and merging supermassive black holes. Monthly Notices of the Royal Astronomical Society 412:1591-1598

[102] Parkinson H, Cole S, Helly J (2008) Generating dark matter halo merger trees. Monthly Notices of the Royal Astronomical Society 383:557-564

[103] Steven (Department of Physics Weinberg UoTaA, Weinberg S (2008) Cosmology. Oxford Univ. Publishing, Oxford

[104] Planck Collaboration, Aghanim N, Akrami Y, Ashdown M, Aumont J, Baccigalupi C, Ballardini M, Banday AJ, Barreiro RB, Bartolo N, Basak S, Battye R, Benabed K, Bernard JP, Bersanelli M, Bielewicz P, Bock JJ, Bond JR, Borrill J, Bouchet FR, Boulanger F, Bucher M, Burigana C, Butler RC, Calabrese E, Cardoso JF, Carron J, Challinor A, Chiang HC, Chluba J, Colombo LPL, Combet C, Contreras D, Crill BP, Cuttaia F, de Bernardis P, de Zotti G, Delabrouille J, Delouis JM, Di Valentino E, Diego JM, Doré O, Douspis M, Ducout A, Dupac X, Dusini S, Efstathiou G, Elsner F, Enßlin TA, Eriksen HK, Fantaye Y, Farhang M, Fergusson J, Fernandez-Cobos R, Finelli F, Forastieri F, Frailis M, Fraisse AA, Franceschi E, Frolov A, Galeotta S, Galli S, Ganga K, Génova-Santos RT, Gerbino M, Ghosh T, González-Nuevo J, Górski KM, Gratton S, Gruppuso A, Gudmundsson JE, Hamann J, Handley W, Hansen FK, Herranz D, Hildebrandt SR, Hivon E, Huang Z, Jaffe AH, Jones WC, Karakci A, Keihänen E, Keskitalo R, Kiiveri K, Kim J, Kisner TS, Knox L, Krachmalnicoff N, Kunz M, Kurki-Suonio H, Lagache G, Lamarre JM, Lasenby A, Lattanzi M, Lawrence CR, Le Jeune M, Lemos P, Lesgourgues J, Levrier F, Lewis A, Liguori M, Lilje PB, Lilley M, Lindholm V, López-Caniego M, Lubin PM, Ma YZ, Macías-Pérez JF, Maggio G, Maino D, Mandolesi N, Mangilli A, Marcos-Caballero A, Maris M, Martin PG, Martinelli M, Martínez-González E, Matarrese S, Mauri N, McEwen JD, Meinhold PR, Melchiorri A, Mennella A, Migliaccio M, Millea M, Mitra S, Miville-Deschênes MA, Molinari D, Montier L, Morgante G, Moss A, Natoli P, Nørgaard-Nielsen HU, Pagano L, Paoletti D, Partridge B, Patanchon G, Peiris HV, Perrotta F, Pettorino V, Piacentini F, Polastri L, Polenta G, Puget JL, Rachen JP, Reinecke M, Remazeilles M, Renzi A, Rocha G, Rosset C, Roudier G, Rubiño-Martín JA, Ruiz-Granados B, Salvati L, Sandri M, Savelainen M, Scott D, Shellard EPS, Sirignano C, Sirri G, Spencer LD, Sunyaev R, Suur-Uski AS, Tauber JA, Tavagnacco D, Tenti M, Toffolatti L, Tomasi M, Trombetti T, Valenziano L, Valiviita J, Van Tent B, Vibert L, Vielva P, 
Villa F, Vittorio N, Wand elt BD, Wehus IK, White M, White SDM, Zacchei A, Zonca A (2018) Planck 2018 results. VI. Cosmological parameters. arXiv e-prints arXiv: 1807.06209

[105] Pontzen A, Tremmel M, Roth N, Peiris HV, Saintonge A, Volonteri M, Quinn T, Governato F (2017) How to quench a galaxy. Monthly Notices of the Royal Astronomical Society 465:547-558

[106] Portegies Zwart SF, Baumgardt H, Hut P, Makino J, McMillan SLW (2004) Formation of massive black holes through runaway collisions in dense young star clusters. Nature 428(6984):724-726

[107] Press WH, Schechter P (1974) Formation of Galaxies and Clusters of Galaxies by Self-Similar Gravitational Condensation. The Astrophysical Journal $187: 425-438$

[108] Quinlan GD (1996) The dynamical evolution of massive black hole binaries I. Hardening in a fixed stellar background. Nature Astronomy 1:35-56

[109] Rasera Y, Teyssier R (2006) The history of the baryon budget. Cosmic logistics in a hierarchical universe. Astronomy and Astrophysics 445(1):1-27

[110] Reines AE, Sivakoff GR, Johnson KE, Brogan CL (2011) An actively accreting massive black hole in the dwarf starburst galaxy Henize2-10. Nature 470:66-68

[111] Reines AE, Greene JE, Geha M (2013) Dwarf Galaxies with Optical Signatures of Active Massive Black Holes. The Astrophysical Journal 775:116

[112] Ricarte A, Tremmel M, Natarajan P, Quinn T (2019) Tracing black hole and galaxy co-evolution in the ROMULUS simulations. Monthly Notices of the Royal Astronomical Society 489(1):802-819

[113] Roedig C, Dotti M, Sesana A, Cuadra J, Colpi M (2011) Limiting eccentricity of subparsec massive black hole binaries surrounded by self-gravitating gas discs. Monthly Notices of the Royal Astronomical Society 415:3033-3041

[114] Sahu N, Graham AW, Davis BL (2019) Black Hole Mass Scaling Relations for Early-type Galaxies. I. $\mathrm{M}_{B H}-\mathrm{M}_{*, s p h}$ and $\mathrm{M}_{B H}-\mathrm{M}_{*, g a l}$. The Astrophysical Journal 876(2): 155

[115] Sahu N, Graham AW, Davis BL (2019) Revealing Hidden Substructures in the $\mathrm{M}_{B H^{-}} \sigma$ Diagram, and Refining the Bend in the L- $\sigma$ Relation. The Astrophysical Journal 887(1):10

[116] Schaye J, Crain RA, Bower RG, Furlong M, Schaller M, Theuns T, Dalla Vecchia C, Frenk CS, McCarthy IG, Helly JC, Jenkins A, Rosas-Guevara YM, White SDM, Baes M, Booth CM, Camps P, Navarro JF, Qu Y, Rahmati A, Sawala T, Thomas PA, Trayford J (2015) The EAGLE project: simulating the evolution and assembly of galaxies and their environments. Monthly Notices of the Royal Astronomical Society 446:521-554

[117] Schramm M, Silverman JD (2013) The Black Hole-Bulge Mass Relation of Active Galactic Nuclei in the Extended Chandra Deep Field-South Survey. The Astrophysical Journal 767:13

[118] Scott N, Graham AW, Schombert J (2013) The Supermassive Black Hole Mass-Spheroid Stellar Mass Relation for Sérsic and Core-Sérsic Galaxies. The Astrophysical Journal 768(1):76 
[119] Sesana A, Khan FM (2015) Scattering experiments meet N-body - I. A practical recipe for the evolution of massive black hole binaries in stellar environments. Monthly Notices of the Royal Astronomical Society 454:L66-L70

[120] Sesana A, Haardt F, Madau P (2006) Interaction of Massive Black Hole Binaries with Their Stellar Environment. I. Ejection of Hypervelocity Stars. The Astrophysical Journal 651:392-400

[121] Sesana A, Barausse E, Dotti M, Rossi EM (2014) Linking the Spin Evolution of Massive Black Holes to Galaxy Kinematics. The Astrophysical Journal 794:104

[122] Sesana A, Shankar F, Bernardi M, Sheth RK (2016) Selection bias in dynamically measured supermassive black hole samples: consequences for pulsar timing arrays. Monthly Notices of the Royal Astronomical Society 463:L6L11

[123] Shankar F, Bernardi M, Sheth RK, Ferrarese L, Graham AW, Savorgnan G, Allevato V, Marconi A, Läsker R, Lapi A (2016) Selection bias in dynamically measured supermassive black hole samples: its consequences and the quest for the most fundamental relation. Monthly Notices of the Royal Astronomical Society 460:3119-3142

[124] Shannon RM, Ravi V, Lentati LT, Lasky PD, Hobbs G, Kerr M, Manchester RN, Coles WA, Levin Y, Bailes M, Bhat NDR, Burke-Spolaor S, Dai S, Keith MJ, Osłowski S, Reardon DJ, van Straten W, Toomey L, Wang JB, Wen L, Wyithe JSB, Zhu XJ (2015) Gravitational waves from binary supermassive black holes missing in pulsar observations. Science 349:1522-1525

[125] Sharma S, Steinmetz M (2005) The Angular Momentum Distribution of Gas and Dark Matter in Galactic Halos. The Astrophysical Journal 628(1):21-44

[126] Sheth RK, Tormen G (1999) Large-scale bias and the peak background split. Monthly Notices of the Royal Astronomical Society 308(1):119-126

[127] Somerville RS, Primack JR (1999) Semi-analytic modelling of galaxy formation: the local Universe. Monthly Notices of the Royal Astronomical Society 310:1087-1110

[128] Springel V, Hernquist L (2002) Cosmological smoothed particle hydrodynamics simulations: the entropy equation. Monthly Notices of the Royal Astronomical Society 333(3):649-664

[129] Taffoni G, Mayer L, Colpi M, Governato F (2003) On the life and death of satellite haloes. Monthly Notices of the Royal Astronomical Society 341:434448

[130] Tinker J, Kravtsov AV, Klypin A, Abazajian K, Warren M, Yepes G, Gottlöber S, Holz DE (2008) Toward a Halo Mass Function for Precision Cosmology: The Limits of Universality. The Astrophysical Journal 688(2):709-728

[131] Tremmel M, Karcher M, Governato F, Volonteri M, Quinn TR, Pontzen A, Anderson L, Bellovary J (2017) The Romulus cosmological simulations: a physical approach to the formation, dynamics and accretion models of SMBHs. Monthly Notices of the Royal Astronomical Society 470:1121-1139 
[132] Tremmel M, Governato F, Volonteri M, Quinn TR, Pontzen A (2018) Dancing to CHANGA: a self-consistent prediction for close SMBH pair formation time-scales following galaxy mergers. Monthly Notices of the Royal Astronomical Society 475:4967-4977

[133] van den Bosch FC, Burkert A, Swaters RA (2001) The angular momentum content of dwarf galaxies: new challenges for the theory of galaxy formation. Monthly Notices of the Royal Astronomical Society 326(3):1205-1215

[134] Vasiliev E (2014) Rates of capture of stars by supermassive black holes in non-spherical galactic nuclei. Classical and Quantum Gravity 31(24):244002

[135] Vasiliev E, Antonini F, Merritt D (2014) The Final-parsec Problem in Nonspherical Galaxies Revisited. The Astrophysical Journal 785:163

[136] Vasiliev E, Antonini F, Merritt D (2015) The Final-parsec Problem in the Collisionless Limit. The Astrophysical Journal 810(1):49

[137] Venemans BP, Walter F, Decarli R, Bañados E, Carilli C, Winters JM, Schuster K, da Cunha E, Fan X, Farina EP, Mazzucchelli C, Rix HW, Weiss A (2017) Copious Amounts of Dust and Gas in a $\mathrm{z}=7.5$ Quasar Host Galaxy. The Astrophysical Journal Letters 851(1):L8

[138] Venemans BP, Walter F, Decarli R, Ferkinhoff C, Weiß A, Findlay JR, McMahon RG, Sutherland WJ, Meijerink R (2017) Molecular Gas in Three z $\sim 7$ Quasar Host Galaxies. The Astrophysical Journal 845(2):154

[139] Venemans BP, Decarli R, Walter F, Bañados E, Bertoldi F, Fan X, Farina EP, Mazzucchelli C, Riechers D, Rix HW, Wang R, Yang Y (2018) Dust Emission in an Accretion-rate-limited Sample of $\mathrm{z} \gtrsim 6$ Quasars. The Astrophysical Journal 866(2):159

[140] Vogelsberger M, Genel S, Springel V, Torrey P, Sijacki D, Xu D, Snyder G, Bird S, Nelson D, Hernquist L (2014) Properties of galaxies reproduced by a hydrodynamic simulation. Nature 509:177-182

[141] Volonteri M, Reines AE (2016) Inferences on the Relations Between Central Black Hole Mass and Total Galaxy Stellar Mass in the High-redshift Universe. The Astrophysical Journal Letters 820:L6

[142] Volonteri M, Lodato G, Natarajan P (2008) The evolution of massive black hole seeds. Monthly Notices of the Royal Astronomical Society 383:10791088

[143] Volonteri M, Dubois Y, Pichon C, Devriendt J (2016) The cosmic evolution of massive black holes in the Horizon-AGN simulation. Monthly Notices of the Royal Astronomical Society 460:2979-2996

[144] Watson WA, Iliev IT, D'Aloisio A, Knebe A, Shapiro PR, Yepes G (2013) The halo mass function through the cosmic ages. Monthly Notices of the Royal Astronomical Society 433(2):1230-1245

[145] Yu Q (2002) Evolution of massive binary black holes. Monthly Notices of the Royal Astronomical Society 331:935-958

[146] Zhang J, Hui L (2006) On Random Walks with a General Moving Barrier. The Astrophysical Journal 641(2):641-646 
[147] Zhang J, Ma CP, Fakhouri O (2008) Conditional mass functions and merger rates of dark matter haloes in the ellipsoidal collapse model. Monthly Notices of the Royal Astronomical Society 387(1):L13-L17

[148] Zhao DH, Jing YP, Mo HJ, Börner G (2003) Mass and Redshift Dependence of Dark Halo Structure. The Astrophysical Journal Letters 597(1):L9-L12

[149] Zhao DH, Jing YP, Mo HJ, Börner G (2009) Accurate Universal Models for the Mass Accretion Histories and Concentrations of Dark Matter Halos. The Astrophysical Journal 707(1):354-369

[150] Zjupa J, Springel V (2017) Angular momentum properties of haloes and their baryon content in the Illustris simulation. Monthly Notices of the Royal Astronomical Society 466(2):1625-1647 